\title{
Non-autonomous stochastic evolution equations and applications to stochastic partial differential equations
}

\author{
MARK C. VERAAR
}

Abstract. In this paper we study the following non-autonomous stochastic evolution equation on a Banach space $E$ :

$$
\left\{\begin{aligned}
\mathrm{d} U(t) & =(A(t) U(t)+F(t, U(t))) \mathrm{d} t+B(t, U(t)) \mathrm{d} W_{H}(t), \quad t \in[0, T], \\
U(0) & =u_{0} .
\end{aligned}\right.
$$

Here, $(A(t))_{t \in[0, T]}$ are unbounded operators with domains $(D(A(t)))_{t \in[0, T]}$ which may be time dependent. We assume that $(A(t))_{t \in[0, T]}$ satisfies the conditions of Acquistapace and Terreni. The functions $F$ and $B$ are nonlinear functions defined on certain interpolation spaces and $u_{0} \in E$ is the initial value. $W_{H}$ is a cylindrical Brownian motion on a separable Hilbert space $H$. We assume that the Banach space $E$ is a UMD space with type 2. Under locally Lipschitz conditions we show that there exists a unique local mild solution of (SE). If the coefficients also satisfy a linear growth condition, then it is shown that the solution exists globally. Under assumptions on the interpolation spaces we extend the factorization method of Da Prato, Kwapień, and Zabczyk, to obtain space-time regularity results for the solution $U$ of (SE). For Hilbert spaces $E$ we obtain a maximal regularity result. The results improve several previous results from the literature. The theory is applied to a second-order stochastic partial differential equation which has been studied by Sanz-Solé and Vuillermot. This leads to several improvements of their result.

\section{Introduction}

Let $E$ be a Banach space and $H$ be a separable Hilbert space. Let $(\Omega, \mathcal{F}, \mathbb{P})$ be a complete probability space with a filtration $\left(\mathcal{F}_{t}\right)_{t \in[0, T]}$. In this paper we study the following stochastic evolution equation on $E$ :

$$
\left\{\begin{aligned}
\mathrm{d} U(t) & =(A(t) U(t)+F(t, U(t))) \mathrm{d} t+B(t, U(t)) \mathrm{d} W_{H}(t), \quad t \in[0, T] \\
U(0) & =u_{0} .
\end{aligned}\right.
$$

Here, the operators $(A(t))_{t \in[0, T]}$ are unbounded and have domains $(D(A(t)))_{t \in[0, T]}$ which may be time dependent. The functions $F:[0, T] \times \Omega \times E \rightarrow E$ and $B:[0, T] \times \Omega \times E \rightarrow \mathcal{B}(H, E)$ are measurable and adapted functions and locally

Mathematics Subject Classification (2000): Primary: 60H15, Secondary: 35R60, 47D06

Keywords: Parabolic stochastic evolution equation, Multiplicative noise, Non-autonomous equations, Mild solution, Variational solution, Type 2, UMD, Stochastic convolution, Factorization method, Space-time regularity, Maximal regularity, $H^{\infty}$-Calculus, Stochastic partial differential equation.

The author is supported by the Alexander von Humboldt foundation. 
Lipschitz in a suitable way. $W_{H}$ is a cylindrical Brownian motion with respect to $\left(\mathcal{F}_{t}\right)_{t \in[0, T]}$ on a separable Hilbert space $H . u_{0}$ is an $\mathcal{F}_{0}$-measurable initial value.

Since the 1970s, the problem (SE) has been studied by many authors. We cannot give a complete description of the literature, but let us give references to some selection of papers.

The method based on monotonicity of operators of [28] has been applied to (SE), for instance, in [25] by Krylov and Rozovskiı̌ and in [41,42] by Pardoux. We will not discuss this method in more detail. For this we refer to the monograph [45] of Rozovskiı̌.

In [17], Dawson used semigroup methods to study (SE) in the autonomous case ( $A$ is constant). This work has been further developed by Da Prato and Zabczyk and their collaborators (cf. $[15,16]$ and references therein). In [51], Seidler considered the non-autonomous case with $D(A(t))$ constant in time. In the above-mentioned works, the authors mainly considered their equation in a Hilbert space $E$. In $[8,9]$ Brzeźniak considered the autonomous case of (SE) in a UMD space $E$ with type 2 space (or even in martingale type 2 spaces $E$ ). This allows one to consider (SE) in $L^{p}$-spaces with $p \in[2, \infty)$. Recently in [37], van Neerven, Weis, and the author considered the autonomous case of (SE) in Banach spaces $E$ which include all $L^{p}$-spaces with $p \in[1, \infty)$. In [56] Zimmerschied and the author studied (SE) with additive noise on a general Banach space, and some parts of the current paper build on these ideas.

There are also many important papers where only $L^{p}$-spaces are considered. Note that all of them always have the restriction that $p \in[2, \infty)$. Let us first mention the works of Krylov and collaborators (see [24] and references therein). In these papers the authors use sophisticated methods from partial differential equations and probability theory to obtain strong space-regularity results for non-autonomous equations. Usually, only second-order equations are considered and the methods are not based on semigroup techniques. We explain some papers which use $L^{p}$-methods and semigroup methods. In the paper of Manthey and Zausinger [33] (also see their references) $L^{p}$-methods and comparison methods are used to obtain global existence results for the case where $F$ is non-necessarily of linear growth. Let us mention that they also allow $D(A(t))$ to depend on time. However, they do not give a systematic study of space-time regularity results. We believe it is important to extend the ideas from [33] to our general framework. This could lead to interesting new global existence results. Also Cerrai [13], Sanz-Solé and Vuillermot [46,47], and Zhang [60] consider $L^{p}$-methods. The papers $[46,47]$ were the starting point of our paper. The equation in $[46,47]$ is a second-order equation with time-dependent boundary conditions. Below, we consider it as our model problem.

In this paper we give a systematic theory for parabolic semi-linear stochastic evolution equations, where $D(A(t))$ depends on time. It seems that such a systematic study is new even in the Hilbert space setting. We study the equation (SE) in a UMD space $E$ with type 2 . This class of spaces includes all $L^{p}$-spaces with $p \in[2, \infty)$. 
Although a stochastic integration theory for processes with values in a general UMD is available [36], we restrict ourselves to spaces with type 2 in order to have a richer class of integrable processes (cf. Proposition 2.8). Note that the theory of [36] was applied in [37] for general UMD spaces, but only for autonomous equations. In order to consider nonautonomous equations, it seems that one needs additional assumptions on $A(t)$, and due to the extra technical difficulties we will not consider this situation here.

Throughout the paper we assume that $(A(t))_{t \in[0, T]}$ satisfies the conditions of Acquistapace and Terreni (AT1) and (AT2) (cf. [2] and Section 2.1 below). These conditions are well understood and widely used in the literature. Let us mention that our results generalize the main setting of $[9,15,51]$ in several ways. To prove regularity of the solution we extend the factorization method of Da Prato, Kwapień, and Zabczyk. This well-known method gives space-time regularity of stochastic convolutions. Compared to the known results, the main difficulty in our version of the factorization method is that $D(A(t))$ is time dependent. For Hilbert space $E$, we obtain a maximal regularity result. This extends the result [16, Theorem 6.14] to the nonautonomous case. The main tool in our approach to maximal regularity is McIntosh's $H^{\infty}$-calculus [34].

To avoid technicalities at this point we will explain one of our main results in a simplified setting. Assume the functions $F$ and $B$ defined on $E$ are Lipschitz uniformly in $[0, T] \times \Omega($ see $(\mathrm{H} 2)$ and $(\mathrm{H} 3)$ in Section 5 where a more general situation is considered). In Section 6 we show that (SE) has a unique mild solution. A strongly measurable and adapted process $U:[0, T] \times \Omega \rightarrow E$ is called a mild solution if for all $t \in[0, T]$, almost surely

$$
U(t)=P(t, 0) u_{0}+P * F(\cdot, U)(t)+P \diamond B(\cdot, U)(t) .
$$

Here $(P(t, s))_{0 \leq s \leq t \leq T}$ denotes the evolution system generated by $(A(t))_{t \in[0, T]}$ and $P * F(t)=\int_{0}^{t} P(t, s) F(s, U(s)) \mathrm{d} s, \quad P \diamond B(t)=\int_{0}^{t} P(t, s) B(s, U(s)) \mathrm{d} W_{H}(s)$.

In Section 5 we also introduce the so-called variational solutions in a general setting and show that they are equivalent to mild solutions.

We state a simplified formulation of one of our main results, Theorem 6.3. The hypothesis (AT1) and (AT2) are introduced in Section 2.1. Hypothesis (H1) $\eta_{0}$ is introduced in Section 4, and Hypotheses (H2) and (H3) can be found in Section 5.

THEOREM 1.1. Assume (AT1), (AT2), (H1) $\eta_{0},(\mathrm{H} 2)$ and $(\mathrm{H} 3)$ with $a=\theta=0$. Let $u_{0}: \Omega \rightarrow E$ be strongly $\mathcal{F}_{0}$ measurable. Then the following assertions hold:

(1) There exists a unique mild solution $U$ of (SE) with paths in $C([0, T] ; E)$ almost surely.

(2) If $u_{0} \in\left(E, D(A(0))_{\eta, 2}\right.$ for some $\eta \in\left[0, \frac{1}{2}\right]$, then for every $\delta, \lambda>0$ with $\delta+\lambda<\eta$ there exists a version of $U$ with paths in $C^{\lambda}\left([0, T] ; \widetilde{E}_{\delta}\right)$. 
Here, $\left(E, D(A(0))_{\eta, 2}\right.$ denotes real interpolation between $E$ and $D(A(0))$. However, one may also take other interpolation spaces. One may think of $\widetilde{E}_{\delta}$ as time-independent version of $\left(E, D(A(t))_{\eta, 2}\right.$ (cf. (H1) $\eta_{\eta_{0}}$ in Section 4).

Actually, in Section 5, we will allow $F$ and $B$ which are defined on suitable interpolation spaces and take values in certain extrapolation spaces. This enables us to consider a larger class of noises. Moreover, in Section 7 we even consider the case that $F$ and $B$ are locally Lipschitz and Theorem 1.1 has a version for locally Lipschitz coefficients (see Theorem 7.2). It is also shown there that if additionally $F$ and $B$ satisfy a linear growth condition as well, then the full statements (1) and (2) of Theorem 1.1 still hold in the locally Lipschitz case.

Our model equation is a problem which has been studied in [46,47]. Here, a secondorder equation with time-dependent boundary conditions is considered. Sanz-Solé and Vuillermot use a version of the factorization methods to obtain existence, uniqueness, and regularity results. Their methods are based on estimates for Green's functions. They also consider two types of variational solutions and mild solutions, and they show that these are all equivalent. We obtain existence, uniqueness, and regularity by applying the above abstract framework. This leads to several improvements of $[46,47]$. For example, our space-time regularity results are better (see Remark 8.3). We also show that our variational and mild solutions coincide with their solution concepts. Our setting seems more robust to adjustments of the equation (see Remark 8.1 and Example 8.9).

The stochastic partial differential equation is:

$$
\begin{aligned}
\mathrm{d} u(t, s)= & A(t, s, D) u(t, s)+f(u(t, s)) \mathrm{d} t \\
& \quad+g(u(t, s)) \mathrm{d} W(t, s), \quad t \in(0, T], s \in S, \\
C(t, s, D) u(t, s)= & 0, \quad t \in(0, T], \quad s \in \partial S \\
u(0, s)= & u_{0}(s), \quad s \in S .
\end{aligned}
$$

Here, $S$ is a bounded domain with boundary of class $C^{2}$ and outer normal vector $n(s)$ in $\mathbb{R}^{n}$, and

$$
\begin{aligned}
& A(t, s, D)=\sum_{i, j=1}^{n} D_{i}\left(a_{i j}(t, s) D_{j}\right)+a_{0}(t, s), \\
& C(t, s, D)=\sum_{i, j=1}^{n} a_{i j}(t, s) n_{i}(s) D_{j},
\end{aligned}
$$

where the coefficients $a_{i j}$ and $a_{0}$ are real valued and smooth and such that $A(t, s, D)$ is uniformly elliptic (cf. Example 8.2). The functions $f$ and $g$ are Lipschitz functions and $u_{0}$ is some $\mathcal{F}_{0}$-measurable initial value. $W$ is a Brownian motion which is white with respect to the time variable and colored with respect to the space variable. More precisely, in Example 8.2, we will assume that the covariance $Q \in \mathcal{B}\left(L^{2}(S)\right)$ of $W(1)$ satisfies $\sqrt{Q} \in \mathcal{B}\left(L^{2}(S), L^{\infty}(S)\right)$. 
In Example 8.2 we will show the following consequence of Theorem 1.1. For details we refer to Section 8 .

(1) Let $p \in[2, \infty)$. If $u_{0} \in L^{p}(S)$ a.s., then there exists a unique mild and variational solution $u$ of (1.1) with paths in $C\left([0, T] ; L^{p}(S)\right)$ a.s. Moreover, $u \in$ $L^{2}\left(0, T ; W^{1,2}(S)\right)$ a.s., where $W^{1,2}(S)=H^{1}(S)$ is the Sobolev space.

(2) If $u_{0} \in C^{1}(\bar{S})$ a.s., then the solution $u$ is in $C^{\lambda}\left([0, T] ; C^{2 \delta}(S)\right)$ for all $\lambda, \delta>0$ such that $\lambda+\delta<\frac{1}{2}$. In particular, $u \in C^{\beta_{1}, \beta_{2}}(\bar{S} \times[0, T])$ for all $\beta_{1} \in(0,1)$ and $\beta_{2} \in\left(0, \frac{1}{2}\right)$.

The definition of a variational solution is given in Section 5 (also see Remark 8.5). The definition of $C^{\beta_{1}, \beta_{2}}$ etc. can be found in Section 7. In Example 8.6 we will also obtain a version of the above result for the case $\sqrt{Q} \in \mathcal{B}\left(L^{2}(S), L^{q}(S)\right)$ for some $q \in(1, \infty)$. In Example 8.9 we show how to obtain a version of the above result for locally Lipschitz coefficients $f$ and $b$.

One can also study partial differential equations driven by multiplicative space-time white noise using (SE). For second-order equations, this is only possible for dimension one, and therefore not very illustrative for our setting. In higher dimensions, this seems to be possible if the order of the operator is larger than the dimension. This has been considered in [37] for the autonomous case (also see [9]). In the non-autonomous setting the case of Dirichlet boundary conditions has been studied in [55, Chapter 8]. Some technical details have to be overcome in order to treat the case of more general boundary conditions. Our results also have interesting consequences for stochastic partial differential equations with boundary noise. This is work in progress [49].

The paper is organized as follows. In Section 2, we discuss the preliminaries on evolution families, $H^{\infty}$-calculus, and stochastic integration theory. In Sections 3 and 4 we study space-time regularity of deterministic and stochastic convolutions, respectively. For this, we extend the factorization method for stochastic convolutions. We also prove a maximal regularity result. The abstract stochastic evolution equation will be given in Section 5. Here, we also introduce variational and mild solutions. In Section 6 we construct a unique mild solution of (SE) by fixed-point methods under Lipschitz conditions on the coefficients. The results are extended to the locally Lipschitz case in Section 7. Finally, in Section 8 we consider the example (1.1).

\section{Preliminaries}

Below, we will use several interpolation methods (cf. [54] for details). Let ( $\left.E_{1}, E_{2}\right)$ be an interpolation couple. For $\eta \in(0,1)$ and $p \in[1, \infty],\left(E_{1}, E_{2}\right)_{\eta, p}$ is the real interpolation space between $E_{1}$ and $E_{2}$. Second, $\left[E_{1}, E_{2}\right]_{\theta}$ is the complex interpolation between $E_{1}$ and $E_{2}$.

We write $a \lesssim_{K} b$ to express that there exists a constant $c$, only depending on $K$, such that $a \leq c b$. We write $a{\sim_{K}}_{b}$ to express that $a \lesssim_{K} b$ and $b \lesssim_{K} a$. If there is no danger of confusion we just write $a \lesssim b$ for convenience. 


\subsection{Parabolic evolution families}

Let $(A(t), D(A(t)))_{t \in[0, T]}$ be a family of closed and densely defined linear operators on a Banach space $E$. Consider the non-autonomous Cauchy problem:

$$
\begin{aligned}
u^{\prime}(t) & =A(t) u(t), \quad t \in[s, T], \\
u(s) & =x
\end{aligned}
$$

We say that $u$ is a classical solution of (2.1) if $u \in C([s, T] ; E) \cap C^{1}((s, T] ; E)$, $u(t) \in D(A(t))$ for all $t \in(s, T], u(s)=x$, and $u^{\prime}(t)=A(t) u(t)$ for all $t \in(s, T]$. We call $u$ a strict solution of (2.1) if $u \in C^{1}([s, T] ; E), u(t) \in D(A(t))$ for all $t \in[s, T], u(s)=x$, and $u^{\prime}(t)=A(t) u(t)$ for all $t \in[s, T]$.

A family of bounded operators $(P(t, s))_{0 \leq s \leq t \leq T}$ on $E$ is called a strongly continuous evolution family if

(1) $P(s, s)=I$ for all $s \in[0, T]$.

(2) $P(t, s)=P(t, r) P(r, s)$ for all $0 \leq s \leq r \leq t \leq T$.

(3) The mapping $\left\{(\tau, \sigma) \in[0, T]^{2}: \sigma \leq \tau\right\} \ni(t, s) \rightarrow P(t, s)$ is strongly continuous.

We say that such a family $(P(t, s))_{0 \leq s \leq t \leq T}$ solves $(2.1)\left(\right.$ on $\left.\left(Y_{s}\right)_{s \in[0, T]}\right)$ if $\left(Y_{s}\right)_{s \in[0, T]}$ are dense subspaces of $E$ such that for all $0 \leq s \leq t \leq T$, we have $P(t, s) Y_{s} \subset Y_{t} \subset$ $D(A(t))$ and the function $t \mapsto P(t, s) x$ is a strict solution of (2.1) for every $x \in Y_{s}$. In this case we say that $(A(t))_{t \in[0, T]}$ generates the evolution family $(P(t, s))_{0 \leq s \leq t \leq T}$.

Well-posedness (i.e. existence, uniqueness, and continuous dependence on initial values from $\left.\left(Y_{S}\right)_{s \in[0, T]}\right)$ of (2.1) is equivalent to the existence and uniqueness of a strongly continuous evolution family that solves $(2.1)$ on $\left(Y_{S}\right)_{s \in[0, T]}$ (see $[39,40]$ and the references therein). In the literature many sufficient conditions for this can be found, both in the hyperbolic and parabolic settings (cf. [2, 6, 29,30,43,52,53,58] and the references therein). In the following, we will recall the parabolic setting of $[2,58]$.

If $E$ is a real Banach space, everything as given in the following, should be understood for the complexification of the objects under consideration. First, we recall some results on sectorial operators.

Assume that for a closed operator $(A, D(A))$, there exist constants $M, w \geq 0$ and $\phi \in(\pi / 2, \pi]$ such that $\Sigma(\phi, w) \subset \rho(A)$ and

$$
\|R(\lambda, A)\| \leq \frac{M}{1+|\lambda-w|}, \quad \lambda \in \Sigma(\phi, w) .
$$

Here $\Sigma(\phi, w)=\{w\} \cup\{\lambda \in \mathbb{C} \backslash\{w\}:|\arg (\lambda-w)| \leq \phi\}$. We denote $A_{w}=A-w$.

It is well known that by (2.2), $A$ generates an analytic semigroup. In this case for $\delta>0$ one can define $\left(-A_{w}\right)^{-\delta} \in \mathcal{B}(E)$ by

$$
\left(-A_{w}\right)^{-\delta}=\frac{1}{2 \pi i} \int_{\Gamma}(w-\lambda)^{-\delta} R(\lambda, A) \mathrm{d} \lambda,
$$


where the contour $\Gamma=\{\lambda: \arg (\lambda-w)= \pm \phi\}$ is orientated counter clockwise (cf. $[6,30,43,52]$ for details). Furthermore, recall that the operator $(w-A)^{\delta}$ is defined as the inverse of $(w-A)^{-\delta}$. For all $\beta>\alpha$,

$$
(E, D(A))_{\beta, \infty} \hookrightarrow(E, D(A))_{\alpha, 1} \hookrightarrow D\left((w-A)^{\alpha}\right) \hookrightarrow(E, D(A))_{\alpha, \infty},
$$

where embedding constants only depend on $\alpha, \beta$ and the constants in (2.2).

As before, let $(A(t), D(A(t)))_{t \in[0, T]}$ be a family of closed and densely defined operators on a Banach space $E$. We will briefly discuss the setting of Acquistapace and Terreni [2]. Note that most of the results below have versions for non-densely defined $A(t)$ as well. In fact they study a slightly more general setting.

Condition (AT) is said to be satisfied if the following two conditions hold:

(AT1) $A(t)$ are linear operators on a Banach space $E$ and there are constants $w \in \mathbb{R}$, $K \geq 0$, and $\phi \in\left(\frac{\pi}{2}, \pi\right)$ such that $\Sigma(\phi, w) \subset \varrho(A(t))$ and for all $\lambda \in \Sigma(\phi, w)$ and $t \in[0, T]$,

$$
\|R(\lambda, A(t))\| \leq \frac{K}{1+|\lambda-w|}
$$

(AT2) There are constants $L \geq 0$ and $\mu, v \in(0,1]$ with $\mu+v>1$ such that for all $\lambda \in \Sigma(\phi, 0)$ and $s, t \in[0, T]$,

$$
\left\|A_{w}(t) R\left(\lambda, A_{w}(t)\right)\left(A_{w}(t)^{-1}-A_{w}(s)^{-1}\right)\right\| \leq L|t-s|^{\mu}(|\lambda|+1)^{-v} .
$$

Below it will be convenient to denote $\kappa_{\mu, v}=\mu+v-1 \in(0,1]$.

These conditions have been extensively studied in the literature, where also many examples can be found. The first condition may be seen as analyticity uniformly in $t \in[0, T]$.

If (AT1) holds and the domains are constant $D(A(0))=D(A(t)), t \in[0, T]$, then Hölder continuity of $(A(t))_{t \in[0, T]}$ in $\mathcal{B}(D(A(0)), E)$ with exponent $\eta$, implies (AT2) with $\mu=\eta$ and $v=1$ (see [2, Section 7]). The conditions in that case reduce to the conditions in the theory of Sobolevskiu and Tanabe for constant domains (cf. [30,43,52]).

We will use the notation $E_{\eta}^{t}=(E, D(A(t)))_{\eta, 2}$ for $t \in[0, T]$ unless it is stated otherwise. Also see $(\mathrm{H} 1)_{\eta_{0}}^{\prime}$ in Section 2.1. Further, we write that $E_{-\theta}^{t}$ for the completion of $E$ with respect to the norm $\|x\|_{E_{-\theta}^{t}}=\left\|\left(-A_{w}(t)\right)^{-\theta} x\right\|$.

Under the assumptions (AT1) and (AT2) the following result holds (see [2, Theorems 6.1-6.4] and [58, Theorem 2.1]):

THEOREM 2.1. If condition (AT) holds, then there exists a unique strongly continuous evolution family $(P(t, s))_{0 \leq s \leq t \leq T}$ that solves $(2.1)$ on $D(A(s))$ and for all $x \in E, P(t, s) x$ is a classical solution of (2.1). Moreover, $(P(t, s))_{0 \leq s \leq t \leq T}$ is continuous on $0 \leq s<t \leq T$ and there exists a constant $C>0$ such that for every $0 \leq s<t \leq T$,

$$
\|P(t, s) x\|_{E_{\alpha}^{t}} \leq C(t-s)^{\beta-\alpha}\|x\|_{E_{\beta}^{s}} \quad \text { for } 0 \leq \beta \leq \alpha \leq 1,
$$


We recall from [58, Theorem 2.1] that there is a constant $C>0$ such that for all $\theta \in(0, \mu)$ and for all $x \in D\left((w-A(s))^{\theta}\right)$,

$$
\left\|P(t, s)(w-A(s))^{\theta} x\right\| \leq C(\mu-\theta)^{-1}(t-s)^{-\theta}\|x\| .
$$

Consider the following Hypothesis.

$(\mathrm{H} 1)_{\eta_{0}}^{\prime}$ There exists an $\eta_{0} \in(0,1]$ and an family of spaces $\left(\widetilde{E}_{\eta}\right)_{\eta \in\left[0, \eta_{0}\right]}$ such that

$$
\widetilde{E}_{\eta_{0}} \hookrightarrow \widetilde{E}_{\eta_{1}} \hookrightarrow \widetilde{E}_{\eta_{2}} \hookrightarrow \widetilde{E}_{0}=E, \quad 0 \leq \eta_{2} \leq \eta_{1} \leq \eta_{0}
$$

and for all $\eta \in\left[0, \eta_{0}\right]$

$$
E_{\eta}^{t}:=(E, D(A(t)))_{\eta, 2} \hookrightarrow \widetilde{E}_{\eta} \hookrightarrow E
$$

with uniform constants in $t \in[0, T]$.

Alternatively, one could replace $(E, D(A(t)))_{\eta, 2}$ by $(E, D(A(t)))_{\eta, p}$ for $p \in(2, \infty)$ or by the complex interpolation spaces $[E, D(A(t))]_{\eta}$.

Assumption $(\mathrm{H} 1)_{\eta_{0}}^{\prime}$ enables us to deduce space-time regularity results. Such type of conditions are often used to get rid of the time dependence. In applications this gives a way to obtain Hölder regularity in space. A similar condition can be found in [32, Hypothesis (H2)]. Later on we will strengthen $(\mathrm{H} 1)_{\eta_{0}}^{\prime}$ to a condition $(\mathrm{H} 1)_{\eta_{0}}$ (see Section 4). There we also require that the space $\widetilde{E}_{\eta}$ are UMD and of type 2 . This is the main reason why one can only allow $p \in[2, \infty)$ if one considers $(E, D(A(t)))_{\eta, p}$.

In many examples one can take $\widetilde{E}_{\eta}=E_{\eta}^{t}$ for $\eta$ small. For second-order operators on $L^{p}$-spaces, $(\mathrm{H} 1)_{\eta_{0}}^{\prime}$ is usually fulfilled for $\eta_{0}=\frac{1}{2}$. However, since it can be difficult to calculate $E_{\eta}^{t}$ it will be convenient to work in the above setting. In the next example we briefly motivate why it is useful to consider the spaces $\widetilde{E}_{\eta}$.

EXAMPLE 2.2. Consider a second-order elliptic differential operator $A(t)$ on a domain $S$ with time-dependent boundary condition $C(t) u=0$. If this is modeled on $E=L^{p}(S)$ for $p \in[2, \infty)$, then one usually has $D(A(t))=\{f \in$ $\left.W^{2, p}(S): C(t) f=0\right\}$. Often one shows that the solution $u$ takes its values in $E_{\eta}^{t}=(E, D(A(t)))_{\eta, 2}$. However, it may be difficult to characterize $E_{\eta}^{t}$ because of the boundary condition. It is even not clear whether such a space is time independent. This will be needed below. It is easier to calculate $\widetilde{E}_{\eta}=\left(E, W^{2, p}(S)\right)_{\eta, 2}$, which is $B_{p, 2}^{2 \eta}(S)$ for regular $S$. This space is time-independent and regularity in the space $B_{p, 2}^{2 \eta}(S)$ usually suffices.

Recall from Grisvard's result (cf. [54, Theorem 4.3.3]) that for domains and coefficients which are $C^{\infty}$ one can characterize the spaces $E_{\eta}^{t}$ as certain subspaces of $B_{p, 2}^{2 \eta}(S)$. A similar result for complex interpolation spaces follows from Seeley [50]. In Amann [5, Section 7] it is explained that for second-order elliptic operators on $L^{p}$-spaces the boundary conditions in $\left.D(A(t))\right)_{\eta, p}$ for $p \in(2, \infty)$ or $[E, D(A(t))]_{\eta}$ disappear under the natural conditions on $p$ and $\eta$. Although his assumptions on the domain seem to be that it is $C^{\infty}$, it follows from [5, Remark 7.3] and [18, Theorem 2.3] that a $C^{2}$ boundary suffices. 
LEMMA 2.3. Assume (AT) and (H1) $\eta_{\eta_{0}}^{\prime}$ for some $\eta_{0} \in(0,1]$.

Let $\alpha \in\left(0, \eta_{0}\right]$. Let $\delta, \lambda>0$ be such that $\delta+\lambda \leq \alpha$. Then there exists a constant $C$ such that for all $0 \leq r \leq s \leq t \leq T$ and for all $x \in E_{\alpha}^{r}$

$$
\|P(t, r) x-P(s, r) x\|_{\widetilde{E}_{\delta}} \leq C|t-s|^{\lambda}\|x\|_{E_{\alpha}^{r}} .
$$

Moreover, if $\alpha \in\left[0, \eta_{0}\right)$ and $x \in E_{\alpha}^{r}$, then $t \mapsto P(t, r) x \in C\left([r, T] ; \widetilde{E}_{\alpha}\right)$.

REMARK 2.4. Under additional assumption on $\alpha, \delta, \mu, v$, there is a version of Lemma 2.3 for the case that $\alpha>\eta_{0}$. Since we will not need this in our examples, we will not consider this situation.

Proof. It follows from (2.4) that

$$
\begin{aligned}
\|P(t, r) x-P(s, r) x\|_{\widetilde{E}_{\alpha}} & \leq\|P(t, r) x\|_{\widetilde{E}_{\alpha}}+\|P(s, r) x\|_{\widetilde{E}_{\alpha}} \\
& \lesssim\|P(t, r) x\|_{E_{\alpha}^{t}}+\|P(s, r) x\|_{E_{\alpha}^{s}} \lesssim\|x\|_{E_{\alpha}^{r}} .
\end{aligned}
$$

Moreover, by $[48,(2.16)]$ we obtain that

$$
\|P(t, r) x-P(s, r) x\| \leq C|t-s|^{\alpha}\|x\|_{E_{\alpha}^{r}} .
$$

Therefore, by interpolation with $\delta=\theta \alpha$ and $\lambda=(1-\theta) \alpha$ for $\theta \in[0,1]$ we obtain

$$
\|P(t, r) x-P(s, r) x\|_{\widetilde{E}_{\delta}} \lesssim|t-s|^{\lambda}\|x\|_{E_{\alpha}^{r}} .
$$

This proves the first part.

For the second part, let $x \in E_{\alpha}^{r}$, and take $x_{1}, x_{2} \ldots$ in $E_{\eta_{0}}^{r}$ such that $x=\lim _{n \rightarrow \infty} x_{n}$ in $E_{\alpha}^{r}$. Then the first result shows that each $t \mapsto P(t, r) x_{n}$ in $\widetilde{E}_{\alpha}$ is continuous. Moreover, as seen earlier,

$$
\left\|P(t, r) x-P(t, r) x_{n}\right\|_{\widetilde{E}_{\alpha}}=\left\|P(t, r)\left(x-x_{n}\right)\right\|_{\widetilde{E}_{\alpha}} \lesssim\left\|x-x_{n}\right\|_{E_{\alpha}^{r}} .
$$

Therefore, $P(t, r) x=\lim _{n \rightarrow \infty} P(t, r) x_{n}$ in $\widetilde{E}_{\alpha}$ uniformly in $t \in[0, T]$, and it is continuous.

\section{2. $H^{\infty}$-Calculus on Hilbert spaces}

In Section 4 we use McIntosh's $H^{\infty}$-calculus in order to derive maximal regularity of stochastic convolutions on Hilbert spaces $E$. Here, we briefly recall the definition and a characterization which gives the way in which we will use the $H^{\infty}$-calculus. For details we refer to $[4,21,26,34]$ and references therein. Although we only explain $H^{\infty}$-calculus on Hilbert spaces, there are extensions to Banach spaces. Our situation slightly differs from the existing literature in the sense that $A$ is replaced by $-A$ and we assume $0 \in \rho(A)$. Moreover, we only consider analytic semigroup generators below.

Let $E$ be a Hilbert space and let $A$ be a closed, densely defined operator on $E$. Assume $A$ is sectorial of type $\phi \in(\pi / 2, \pi]$, i.e., the sector $\Sigma_{\phi}:=\Sigma(\phi, 0) \subset \rho(A)$ and there exists a constant $M$ such that for all

$$
\|R(\lambda, A)\| \leq \frac{M}{1+|\lambda|}, \quad \lambda \in \Sigma_{\phi}
$$

The largest constant $\phi$ for which such an $M$ exists will be denoted with $\phi(A)$. 
For $\sigma \in(0, \pi]$, let $H^{\infty}\left(\Sigma_{\sigma}\right)$ denote the space of bounded analytic functions $f$ : $\Sigma_{\sigma} \rightarrow \mathbb{C}$ with norm $\|f\|_{H^{\infty}\left(\Sigma_{\sigma}\right)}=\sup _{\lambda \in \Sigma_{\sigma}}|f(\lambda)|$. Let

$$
H_{0}^{\infty}\left(\Sigma_{\sigma}\right)=\left\{f \in H^{\infty}\left(\Sigma_{\sigma}\right): \exists \epsilon>0 \text { s.t. }|f(\lambda)| \leq \frac{|z|^{\varepsilon}}{\left(1+|z|^{2}\right)^{\varepsilon}}\right\} .
$$

Let $A$ be as above, and fix $\sigma \in(\pi / 2, \phi(A))$ and $\theta \in(\sigma, \phi(A))$. For $f \in H_{0}^{\infty}\left(\Sigma_{\sigma}\right)$, one can define

$$
f(A)=\frac{1}{2 \pi i} \int_{\partial \Sigma_{\theta}} f(\lambda) R(\lambda, A) \mathrm{d} \lambda
$$

where the integral converges in the Bochner sense. We say that $A$ has a bounded $H^{\infty}\left(\Sigma_{\sigma}\right)$-calculus for $\sigma \in(\pi / 2, \phi(A))$ if there is a constant $C$ such that

$$
\|f(A)\| \leq C\|f\|_{H^{\infty}\left(\Sigma_{\sigma}\right)} \text { for all } f \in H_{0}^{\infty}\left(\Sigma_{\sigma}\right) .
$$

In this case, (2.7) has a unique continuous extension to all $f \in H^{\infty}\left(\Sigma_{\sigma}\right)$. The boundedness of the $H^{\infty}$-calculus is characterized by the following theorem:

PROPOSITION 2.5. Let $E$ be a Hilbert space and let $A$ be as above. Then the following assertions are equivalent:

(1) A has a bounded $H^{\infty}\left(\Sigma_{\sigma}\right)$-calculus for some (all) $\sigma \in(\pi / 2, \phi(A))$.

(2) -A has bounded imaginary powers and for some (all) $\sigma \in(\pi / 2, \phi(A))$ there is a constant $C>0$ such that for all $s \in \mathbb{R},\left\|(-A)^{i s}\right\| \leq C \mathrm{e}^{\sigma|s|}$.

(3) For some (all) $|\sigma| \in(-\phi(A), \phi(A))$ there exists a constant $C>0$ such that for all $x \in E$,

$$
C^{-1}\|x\| \leq\left(\int_{0}^{\infty}\left\|(-A)^{\frac{1}{2}} R\left(t \mathrm{e}^{i \sigma}, A\right) x\right\|^{2} \mathrm{~d} t\right)^{\frac{1}{2}} \leq C\|x\| .
$$

This result can be found in [34] (also see [26, Theorem 11.9]). The estimate (2.8) is called a square function estimate. Applying the Fourier transform to $t \mapsto R\left(t \mathrm{e}^{i \sigma}, A\right) x$ with $\sigma=\pi / 2$ one obtains that there exists a constant $C_{2}$ such that for all $x \in E, \mathrm{C}$

$$
C_{2}^{-1}\|x\| \leq\left(\int_{0}^{\infty}\left\|(-A)^{\frac{1}{2}} \mathrm{e}^{t A} x\right\|^{2} \mathrm{~d} t\right)^{\frac{1}{2}} \leq C_{2}\|x\| . C
$$

The important estimate for us will be

$$
\left(\int_{0}^{\infty}\left\|(-A)^{\frac{1}{2}} \mathrm{e}^{t A}\right\|^{2} \mathrm{~d} t\right)^{\frac{1}{2}} \leq C_{2}\|x\| .
$$

The same estimates as in (2.10) hold for $A^{*}$. Moreover, if (2.10) holds for $A$ and $A^{*}$, this again implies the boundedness of the $H^{\infty}$-calculus. We further note that the estimate (2.10) is also used for the Weiss conjecture in control theory (cf. [27] and references therein). 
Not every sectorial operator $A$ has a bounded $H^{\infty}$-calculus of some angle. Counterexamples are given in [35]. However, many examples are known to have a bounded $H^{\infty}$-calculus. We state some sufficient conditions for the boundedness of the $H^{\infty}$ calculus.

REMARK 2.6. For a Hilbert space $E$, each of the following conditions is sufficient for having a bounded $H^{\infty}$-calculus:

(1) A generates an analytic contraction semigroup (see [26, Theorem 11.13]).

(2) $-A$ is positive and self-adjoint. In this case one has $C_{2}=1$ in (2.9) (cf. [26, Example 11.7]) and $C=1$ in (2.7) (cf. [4, Section (G)]).

\section{3. $\gamma$-Radonifying operators and stochastic integration}

We recall some results on $\gamma$-radonifying operators and stochastic integration. For details on the subject we refer to [7,9,20,22,38,36].

Let $E$ be a Banach space and $H$ be a separable Hilbert space. Let $(S, \mu)$ be a measurable space. A function $\phi: S \rightarrow E$ is called strongly measurable if it is the pointwise limit of a sequence of simple functions.

Let $E_{1}$ and $E_{2}$ be Banach spaces. An operator-valued function $\Phi: S \rightarrow \mathcal{B}\left(E_{1}, E_{2}\right)$ will be called $E_{1}$-strongly measurable if for all $x \in E_{1}$, the $E_{2}$-valued function $\Phi x$ is strongly measurable.

If $(S, \Sigma, \mu)$ is a measure space and $\phi: S \rightarrow E$ is defined as an equivalence class of functions, then we say that $\phi$ is strongly measurable if there is a version of $\phi$ which is strongly measurable.

A bounded operator $R \in \mathcal{B}(H, E)$ is said to be a $\gamma$-radonifying operator if there exists an orthonormal basis $\left(h_{n}\right)_{n \geq 1}$ of $H$ such that $\sum_{n \geq 1} \gamma_{n} R h_{n}$ converges in $L^{2}(\Omega ; E)$. We then define

$$
\|R\|_{\gamma(H, E)}:=\left(\mathbb{E}\left\|\sum_{n \geq 1} \gamma_{n} R h_{n}\right\|^{2}\right)^{\frac{1}{2}} .
$$

This number does not depend on the sequence $\left(\gamma_{n}\right)_{n \geq 1}$ and the basis $\left(h_{n}\right)_{n \geq 1}$, and defines a norm on the space $\gamma(H, E)$ of all $\gamma$-radonifying operators from $H$ into $E$. Endowed with this norm, $\gamma(H, E)$ is a Banach space, which is separable if $E$ is separable. Moreover, $\|R\| \leq\|R\|_{\gamma(H, E)}$.

If $E$ is a Hilbert space, then $\gamma(H, E)=\mathcal{C}^{2}(H, E)$ isometrically, where $\mathcal{C}^{2}(H, E)$ is the space of Hilbert-Schmidt operators. Also for $E=L^{p}$ there are easy characterization of $\gamma$-radonifying operators. Such a characterization has been obtained in [11]. We use a slightly different formulation from [37].

LEMMA 2.7. Let $(S, \Sigma, \mu)$ be a $\sigma$-finite measure space and let $1 \leq p<\infty$. For an operator $R \in \mathcal{B}\left(H, L^{p}(S)\right)$ the following assertions are equivalent: 
(1) $R \in \gamma\left(H, L^{p}(S)\right)$.

(2) There exists a function $g \in L^{p}(S)$ such that for all $h \in H$ we have $|R h| \leq$ $\|h\|_{H} \cdot g \mu$-almost everywhere.

Moreover, in this situation we have

$$
\|R\|_{\gamma\left(H, L^{p}(S)\right)} \lesssim p\|g\|_{L^{p}(S)} .
$$

Let $\left(r_{n}\right)_{n \geq 1}$ be a Rademacher sequence on $(\Omega, \mathcal{F}, \mathbb{P})$. Recall that a Banach space $E$ is said to have type 2 if there exists a constant $C \geq 0$ such that for all finite subsets $\left\{x_{1}, \ldots, x_{N}\right\}$ of $E$ we have

$$
\left(\mathbb{E}\left\|\sum_{n=1}^{N} r_{n} x_{n}\right\|^{2}\right)^{\frac{1}{2}} \leq C\left(\sum_{n=1}^{N}\left\|x_{n}\right\|^{2}\right)^{\frac{1}{2}} .
$$

Hilbert spaces have type 2 and the $L^{p}$-spaces for $p \in[2, \infty)$ have type 2 (see [20, Chapter 11] for details). Furthermore, Besov spaces $B_{p, q}^{\alpha}$ and Sobolev spaces $W^{\alpha, p}$ have type 2 as long as $2 \leq p, q<\infty$. This follows from the fact that these spaces are isomorphic to closed subspaces of $L^{p}$-spaces and $\ell^{q}\left(L^{p}\right)$-spaces (cf. [54]).

We will also need UMD Banach spaces. The definition of a UMD space will be omitted. We refer to [12] for an overview on the subject. Important examples of UMD spaces are the reflexive scale of $L^{p}$, Sobolev and Besov spaces. Moreover, we note that every UMD space is reflexive.

A detailed stochastic integration theory for operator-valued processes $\Phi:[0, T] \times$ $\Omega \rightarrow \mathcal{B}(H, E)$ where $E$ is a UMD space, is given in [36]. For our purposes it will be enough to consider UMD spaces with type 2 . In this situation there is an easy subspace of integrable processes which will be large enough for our considerations. Instead of UMD spaces with type 2, one can also assume that $E$ is a of martingale type 2 space (cf. $[9,44]$ ). We do not consider this generality, because it is unnecessary for our applications to stochastic partial differential equations.

A family $W_{H}=\left(W_{H}(t)\right)_{t \in \mathbb{R}_{+}}$of bounded linear operators from $H$ to $L^{2}(\Omega)$ is called an $H$-cylindrical Brownian motion if

(i) $W_{H} h=\left(W_{H}(t) h\right)_{t \in \mathbb{R}_{+}}$is a scalar-valued Brownian motion for each $h \in H$,

(ii) $\mathbb{E}\left(W_{H}(s) g \cdot W_{H}(t) h\right)=(s \wedge t)[g, h]_{H}$ for all $s, t \in \mathbb{R}_{+}, g, h \in H$.

We always assume that the $H$-cylindrical Brownian motion $W_{H}$ is with respect to the filtration $\left(\mathcal{F}_{t}\right)_{t \geq 0}$, i.e., $W_{H} h$ are Brownian motions with respect to $\left(\mathcal{F}_{t}\right)_{t \geq 0}$ for all $h \in H$.

Now let $E$ be a UMD Banach space with type 2. For an $H$-strongly measurable and adapted $\Phi:[0, T] \times \Omega \rightarrow \gamma(H, E)$ which is in $L^{2}((0, T) \times \Omega ; \gamma(H, E))$ one can define the stochastic integral $\int_{0}^{T} \Phi(s) \mathrm{d} W_{H}(s)$ as a limit of integrals of adapted step processes and (cf. [9] and references therein) there exists a constant $C$ not depending on $\Phi$ such that

$$
\left(\mathbb{E}\left\|\int_{0}^{T} \Phi(s) \mathrm{d} W_{H}(s)\right\|^{2}\right)^{\frac{1}{2}} \leq C\|\Phi\|_{L^{2}((0, T) \times \Omega ; \gamma(H, E))} .
$$


By a localization argument one may extend the class of integrable processes to all $H$-strongly measurable and adapted $\Phi:[0, T] \times \Omega \rightarrow \gamma(H, E)$ which are in $L^{2}(0, T ; \gamma(H, E))$ a.s. Moreover, the estimate (2.13) for the stochastic integral also implies type 2 .

In [36] two-sided estimates for the stochastic integral are given using generalized square function norms. As a consequence of that theory one also obtains the above results. The result that we will frequently use is the following (cf. [9] and [36, Corollary 3.10]).

PROPOSITION 2.8. Let $E$ be a UMD space with type 2. Let $\Phi:[0, T] \times \Omega \rightarrow$ $\gamma(H, E)$ be $H$-strongly measurable and adapted. If $\Phi \in L^{2}(0, T ; \gamma(H, E))$ a.s., then $\Phi$ is stochastically integrable with respect to $W_{H}$ and for all $p \in(1, \infty)$,

$$
\left(\mathbb{E} \sup _{t \in[0, T]}\left\|\int_{0}^{t} \Phi(s) d W_{H}(s)\right\|^{p}\right)^{\frac{1}{p}} \lesssim_{E, p}\|\Phi\|_{L^{p}\left(\Omega ; L^{2}(0, T ; \gamma(H, E))\right)} .
$$

Again the estimate in Proposition 2.8 implies that $E$ has type 2.

We will also use the following basic fact for $\Phi$ : as in Proposition 2.8 for $x^{*} \in E^{*}$,

$$
\left\langle\int_{0}^{T} \Phi(s) \mathrm{d} W_{H}(s), x^{*}\right\rangle=\int_{0}^{T} \Phi(s)^{*} x^{*} \mathrm{~d} W_{H}(s) \text { a.s. }
$$

\section{Deterministic convolutions}

Let $E$ be a Banach space. For $\alpha \in(0,1], p \in[1, \infty]$, and $f \in L^{p}(0, T ; E)$, define the function $R_{\alpha} f \in L^{p}(0, T ; E)$ by

$$
\left(R_{\alpha} f\right)(t)=\frac{1}{\Gamma(\alpha)} \int_{0}^{t}(t-s)^{\alpha-1} P(t, s) f(s) \mathrm{d} s .
$$

This is well defined by Young's inequality, and there is a constant $C \geq 0$ that only depends on $\alpha, p$ and $\sup _{0 \leq s \leq t \leq T}\|P(t, s)\|$ such that

$$
\left\|R_{\alpha} f\right\|_{L^{p}(0, T ; E)} \leq C T^{\alpha}\|f\|_{L^{p}(0, T ; E)} .
$$

LEMMA 3.1. Assume that (AT) and (H1) $\eta_{0}^{\prime}$ with some $\eta_{0} \in(0,1]$ hold. Let $\alpha \in$ $\left(0, \eta_{0}\right], \delta, \lambda \in[0,1)$, and $p \in[1, \infty)$ be such that $\alpha-\frac{1}{p}-\delta-\lambda>0$. Then for every $f \in L^{p}(0, T ; E), R_{\alpha} f \in C^{\lambda}\left([0, T] ; \widetilde{E}_{\delta}\right)$ and there is a constant $C \geq 0$ such that for all $f \in L^{p}(0, T ; E)$,

$$
\left\|R_{\alpha} f\right\|_{C^{\lambda}\left([0, T] ; \widetilde{E}_{\delta}\right)} \leq C\|f\|_{L^{p}(0, T ; E)} .
$$

Proof. This can be proved in a similar way as in [56, Lemma 4.1], by replacing the fractional domain spaces by $\widetilde{E}_{\eta}$. The only part of the proof of [56, Lemma 4.1] that requires a different argument is the estimate for

$$
I_{3}=\left\|\int_{0}^{s}(s-r)^{\alpha-1}(P(t, r)-P(s, r)) f(r) \mathrm{d} r\right\|_{\widetilde{E}_{\delta}} .
$$


We have to show that $I_{3} \lesssim|t-s|^{\lambda}$. It follows from Lemma 2.3 and 2.4 that for $x \in E$

$$
\begin{aligned}
\|P(t, r) x-P(s, r) x\|_{\widetilde{E}_{\delta}} & =\|(P(t, s)-I) P(s, r) x\|_{\widetilde{E}_{\delta}} \\
& \lesssim|t-s|^{\lambda}\|P(s, r) x\|_{\widetilde{E}_{\delta+\lambda}} \\
& \lesssim|t-s|^{\lambda}\|P(s, r) x\|_{E_{\delta+\lambda}^{r}} \\
& \lesssim|t-s|^{\lambda}(s-r)^{-\lambda-\delta}\|x\| .
\end{aligned}
$$

This implies the estimate for $I_{3}$.

Recall that $E_{-\theta}^{t}$ be the completion of $E$ with respect to the norm $\|x\|_{E_{-\theta}^{t}}=$ $\left\|\left(-A_{w}(t)\right)^{-\theta} x\right\|$.

The next result will be formulated for a family $\{\phi(t): t \in[0, T]\}$ such that for all $t \in[0, T], \phi(t, \omega) \in E_{-\theta}^{t}$, where $\left(-A_{w}\right)^{-\theta} \phi$ is a strongly measurable function from $[0, T]$ into $E$ and $\theta \in[0,1)$ is fixed. We denote the deterministic convolution by

$$
P * \Phi(t):=\int_{0}^{t} P(t, s) \phi(s) \mathrm{d} s
$$

where $\phi$ is as above.

First, we explain some general measurability properties which hold under the (AT) conditions. Let $\theta \in[0, \mu)$. One has that for all $0 \leq s<t \leq T, P(t, s)(w-A(s))^{\theta}$ has an extension to an operator in $\mathcal{B}(E)$ (see (2.5)). We claim that as a function of $(s, t)$ where $0 \leq s<t \leq T$, this extension is $E$-strongly measurable.

Indeed, let $A_{n}(t)=n A(t) R(n ; A(t))$ be the Yosida approximations of $A(t)$. Then for all $x \in E$ (see proof of [57, Proposition 3.1]) one has

$$
\lim _{n \rightarrow \infty} P_{n}(t, s)\left(w-A_{n}(s)\right)^{\theta} x=P(t, s)(w-A(s))^{\theta} x,
$$

where $P_{n}(t, s)$ is the evolution family generated by $A_{n}(t)$. Since $(t, s) \mapsto P_{n}(t, s)(w-$ $\left.A_{n}(s)\right)^{\theta} x$ is strongly measurable, the claim follows.

It follows that for $0 \leq s<t \leq T, P(t, s)$ has a unique extension to an operator in $\mathcal{B}\left(E_{-\theta}^{s}, E\right)$. We will denote this extension again by $P(t, s)$. Below we will need to integrate $P(t, s) \phi(s)$ with respect to $\mathrm{d} s$. This can be made rigorous in the same way as in [31] using the extension of $P(t, s)$ to $\mathcal{B}\left(E_{-\theta}^{s}, E\right)$. If $\phi$ is as above and $\left(-A_{w}(\cdot)\right)^{-\theta} \phi \in L^{p}(0, T ; E)$ one could equivalently say $\phi \in X_{-\theta}$ a.s., where $X=L^{p}(0, T ; E)$ and $X_{-\theta}$ is the extrapolation space under $A_{w}(\cdot)$ as a sectorial operator on $L^{p}(0, T ; E)$. Below we will not explicitly use the extrapolation spaces and just interpret $P(t, s) \phi(s)$ as $P(t, s)\left(-A_{w}(s)\right)^{\theta}\left(-A_{w}(s)\right)^{-\theta} \phi(s)$. This is allowed since for $x \in E_{-\theta}^{s}$ it is easily checked that

$$
P(t, s) x=P(t, s)\left(-A_{w}(s)\right)^{\theta}\left(-A_{w}(s)\right)^{-\theta} x .
$$


PROPOSITION 3.2. Assume that (AT) and (H1) $\eta_{\eta_{0}}^{\prime}$ hold. Let $\theta \in[0, \mu)$ Let $p \in$ $(1, \infty], \delta \in[0,1)$ and $\lambda \in(0,1)$ be such that $\lambda+\delta+\frac{1}{p}<\min \left\{1-\theta, \eta_{0}\right\}$. Then there exists a constant $C_{T}$ with $\lim _{T \downarrow 0} C_{T}=0$ such that for all $\left(-A_{w}\right)^{-\theta} \phi \in L^{p}(0, T ; E)$,

$$
\|P * \phi\|_{C^{\lambda}\left([0, T] ; \widetilde{E}_{\delta}\right)} \leq C_{T}\left\|\left(-A_{w}\right)^{-\theta} \phi\right\|_{L^{p}(0, T ; E)} .
$$

Proof. First note that

$$
\begin{aligned}
& \{(t, s): 0 \leq s<t \leq T\} \ni(t, s) \mapsto P(t, s) \phi(s) \\
& \quad=P(t, s)\left(-A_{w}(s)\right)^{\theta}\left(-A_{w}(s)\right)^{-\theta} \phi(s)
\end{aligned}
$$

is a strongly measurable $E$-valued function.

Let $\alpha>0$ be such that $\lambda+\delta+\frac{1}{p}<\alpha<\min \left\{1-\theta, \eta_{0}\right\}$. Define $\zeta_{\alpha}:[0, T] \rightarrow E$ as

$$
\zeta_{\alpha}(t)=\frac{1}{\Gamma(1-\alpha)} \int_{0}^{t}(t-s)^{-\alpha} P(t, s) \phi(s) \mathrm{d} s .
$$

Then by (2.5), for each $t \in[0, T]$,

$$
\begin{aligned}
\left\|\zeta_{\alpha}(t)\right\| & \leq \frac{1}{\Gamma(1-\alpha)} \int_{0}^{t}(t-s)^{-\alpha}\|P(t, s) \phi(s)\| \mathrm{d} s \\
& \lesssim \int_{0}^{t}(t-s)^{-\alpha-\theta}\left\|\left(-A_{w}(s)\right)^{-\theta} \phi(s)\right\| \mathrm{d} s .
\end{aligned}
$$

Therefore, by Young's inequality

$$
\begin{aligned}
\left\|\zeta_{\alpha}\right\|_{L^{p}(0, T ; E)}^{p} & \lesssim \int_{0}^{T}\left|\int_{0}^{t}(t-s)^{-\alpha-\theta}\left\|\left(-A_{w}(s)\right)^{-\theta} \phi(s)\right\| \mathrm{d} s\right|^{p} \mathrm{~d} t \\
& \leq C_{T}^{p}\left\|\left(-A_{w}(s)\right)^{-\theta} \phi\right\|_{L^{p}(0, T ; E)}^{p} .
\end{aligned}
$$

Define $\zeta:[0, T] \rightarrow E$ as $\zeta=P * \phi$. By Hölders's inequality and $\theta<1-\frac{1}{p}$ this is well defined. We claim that $\zeta=R_{\alpha}\left(\zeta_{\alpha}\right)$. This would complete the proof by Lemma 3.1 and

$$
\begin{aligned}
\|\zeta\|_{C^{\lambda}\left([0, T] ; \widetilde{E}_{\delta}\right)} & =\left\|R_{\alpha}\left(\zeta_{\alpha}\right)\right\|_{C^{\lambda}\left([0, T] ; \widetilde{E}_{\delta}\right)} \\
& \lesssim C_{T}\left\|\zeta_{\alpha}\right\|_{L^{p}(0, T ; E)} \lesssim C_{T}\left\|\left(-A_{w}\right)^{-\theta} \phi\right\|_{L^{p}(0, T ; E)}
\end{aligned}
$$

To prove the claim notice that by Fubini's theorem for all $t \in[0, T]$,

$$
\begin{aligned}
R_{\alpha}\left(\zeta_{\alpha}\right) & =\frac{1}{\Gamma(\alpha)} \int_{0}^{t}(t-s)^{\alpha-1} P(t, s) \zeta_{\alpha}(s) \mathrm{d} s \\
& =\frac{1}{\Gamma(1-\alpha) \Gamma(\alpha)} \int_{0}^{t} \int_{0}^{s}(t-s)^{\alpha-1}(s-r)^{-\alpha} P(t, r) \phi(r) \mathrm{d} r \mathrm{~d} s \\
& =\frac{1}{\Gamma(1-\alpha) \Gamma(\alpha)} \int_{0}^{t} \int_{r}^{t}(t-s)^{\alpha-1}(s-r)^{-\alpha} P(t, r) \phi(r) \mathrm{d} s \mathrm{~d} r \\
& =\int_{0}^{t} P(t, r) \phi(r) \mathrm{d} r=\zeta(t) .
\end{aligned}
$$




\section{Stochastic convolutions}

Let $(\Omega, \mathcal{F}, \mathbb{P})$ be a complete probability space with a filtration $\left(\mathcal{F}_{t}\right)_{t \in[0, T]}$. Let $E$ be a Banach space and $H$ be a separable Hilbert space. Let $W_{H}$ be a cylindrical Wiener process with respect to $\left(\mathcal{F}_{t}\right)_{t \in[0, T]}$. We strengthen the hypothesis $(\mathrm{H} 1)_{\eta_{0}}^{\prime}$ from Section 2.1.

$(\mathrm{H} 1)_{\eta_{0}}$ There exists an $\eta_{0} \in(0,1]$ and a family of spaces $\left(\widetilde{E}_{\eta}\right)_{\eta \in\left[0, \eta_{0}\right]}$ such that each $\widetilde{E}_{\eta}$ is a UMD spaces with type 2 ,

$$
\widetilde{E}_{\eta_{0}} \hookrightarrow \widetilde{E}_{\eta_{1}} \hookrightarrow \widetilde{E}_{\eta_{2}} \hookrightarrow \widetilde{E}_{0}=E, \quad 0 \leq \eta_{2} \leq \eta_{1} \leq \eta_{0}
$$

and for all $\eta \in\left[0, \eta_{0}\right]$

$$
(E, D(A(t)))_{\eta, 2} \hookrightarrow \widetilde{E}_{\eta} \hookrightarrow E
$$

with uniform constants in $t \in[0, T]$.

The next result will be formulated for a family $\{\Phi(t, \omega): t \in[0, T], \omega \in \Omega\}$ such that for all $t \in[0, T]$ and all $\omega \in \Omega, \Phi(t, \omega) \in \mathcal{B}\left(H, E_{-\theta}^{t}\right)$, where $\left(-A_{w}\right)^{-\theta} \Phi$ is an $H$-strongly measurable and adapted process from $[0, T] \times \Omega$ into $\mathcal{B}(H, E)$ and $\theta \in\left[0, \frac{1}{2}\right)$ is fixed. We denote the stochastic convolution by

$$
P \diamond \Phi(t):=\int_{0}^{t} P(t, s) \Phi(s) \mathrm{d} W_{H}(s),
$$

where $\Phi$ is as above. The following extends results from $[9,14,51]$.

THEOREM 4.1. Assume (AT) and (H1) $\eta_{0}$. Let $\theta \in\left[0, \mu \wedge \frac{1}{2}\right)$. Let $p \in(2, \infty)$ and let $\delta, \lambda>0$ be such that $\delta+\lambda+\frac{1}{p}<\min \left\{\frac{1}{2}-\theta, \eta_{0}\right\}$. Let $\left(-A_{w}\right)^{-\theta} \Phi:[0, T] \times$ $\Omega \rightarrow \gamma(H, E)$ be $H$-strongly measurable and adapted such that $\left(-A_{w}\right)^{-\theta} \Phi \in$ $L^{p}(0, T ; \gamma(H, E))$ a.s. Then for all $t \in[0, T], s \mapsto P(t, s) \Phi(s) \in \gamma(H, E)$ is $H$-strongly measurable and adapted, $P \diamond \Phi$ exists in $\widetilde{E}_{\delta}$ and is $\lambda$-Hölder continuous and there exists a constant $C \geq 0$ independent of $\Phi$ such that

$$
\mathbb{E}\|P \diamond \Phi\|_{C^{\lambda}\left([0, T] ; \widetilde{E}_{\delta}\right)}^{p} \leq C \mathbb{E}\left\|\left(-A_{w}\right)^{-\theta} \Phi\right\|_{L^{p}(0, T ; \gamma(H, E))}^{p} .
$$

Proof. We claim that

$$
\{(t, s): 0 \leq s<t \leq T\} \ni(t, s) \mapsto P(t, s) \Phi(s) \in \gamma(H, E)
$$

is $H$-strongly measurable and for all $t \in[0, T]$ and

$$
(0, t) \ni s \mapsto P(t, s) \Phi(s) \in \gamma(H, E)
$$

is $H$-strongly adapted. Indeed, this follows from the assumption and the remarks before Proposition 3.2 as soon as we write

$$
P(t, s) \Phi(s)=P(t, s)(w-A(s))^{\theta}(w-A(s))^{-\theta} \Phi(s) .
$$


Let $\delta$ and $\lambda$ be as in the theorem and let $\alpha$ be such that $\delta+\lambda+\frac{1}{p}<\alpha<\min \left\{\frac{1}{2}-\theta, \eta_{0}\right\}$. Define $\zeta_{\alpha}:[0, T] \times \Omega \rightarrow E$ as

$$
\zeta_{\alpha}(t)=\frac{1}{\Gamma(1-\alpha)} \int_{0}^{t}(t-s)^{-\alpha} P(t, s) \Phi(s) \mathrm{d} W_{H}(s) .
$$

Then by Proposition 2.8, (2.5), Young's inequality and [37, Appendix], $\zeta_{\alpha}$ is well-defined in $L^{p}((0, T) \times \Omega ; E)$ and jointly measurable, and moreover we have

$$
\begin{aligned}
\left\|\zeta_{\alpha}\right\|_{L^{p}((0, T) \times \Omega ; E)} & \lesssim\left(\mathbb{E} \int_{0}^{T}\left(\int_{0}^{t}\left\|(t-s)^{-\alpha} P(t, s) \Phi(s)\right\|_{\gamma(H, E)}^{2} \mathrm{~d} s\right)^{\frac{p}{2}} \mathrm{~d} t\right)^{\frac{1}{p}} \\
& \lesssim\left(\mathbb{E} \int_{0}^{T}\left(\int_{0}^{t}(t-s)^{-2 \alpha-2 \theta}\left\|\left(-A_{w}(s)\right)^{-\theta} \Phi(s)\right\|_{\gamma(H, E)}^{2} \mathrm{~d} s\right)^{\frac{p}{2}} \mathrm{~d} t\right)^{\frac{1}{p}} \\
& \leq C\left(\mathbb{E}\left\|\left(-A_{w}\right)^{-\theta} \Phi\right\|_{L^{p}(0, T ; \gamma(H, E))}^{p}\right)^{\frac{1}{p}} .
\end{aligned}
$$

Here we used $\alpha<\frac{1}{2}-\theta$. Let $\Omega_{0}$ with $P\left(\Omega_{0}\right)=1$ be such that $\zeta_{\alpha}(\cdot, \omega) \in L^{p}(0, T ; E)$ for all $\omega \in \Omega_{0}$. We may apply Lemma 3.1 to obtain that for all $\omega \in \Omega_{0}$,

$$
R_{\alpha} \zeta_{\alpha}(\cdot, \omega) \in C^{\lambda}\left([0, T] ; \widetilde{E}_{\delta}\right)
$$

and

$$
\left\|R_{\alpha} \zeta_{\alpha}(\cdot, \omega)\right\|_{C^{\lambda}\left([0, T] ; \widetilde{E}_{\delta}\right)} \lesssim C\left\|\zeta_{\alpha}(\cdot, \omega)\right\|_{L^{p}(0, T ; E)}
$$

Define $\zeta:[0, T] \times \Omega \rightarrow E$ as $\zeta=P \diamond \Phi$. Since $\theta<\frac{1}{2}-\frac{1}{p}$, one may check that this is well defined. We claim that for all $t \in[0, T]$, for almost all $\omega \in \Omega$, we have

$$
\zeta(t, \omega)=\left(R_{\alpha} \zeta_{\alpha}(\cdot, \omega)\right)(t) .
$$

It suffices to check that for all $t \in[0, T]$ and $x^{*} \in E^{*}$, almost surely we have

$$
\left\langle\zeta(t), x^{*}\right\rangle=\frac{1}{\Gamma(\alpha)} \int_{0}^{t}(t-s)^{\alpha-1}\left\langle P(t, s) \zeta_{\alpha}(s), x^{*}\right\rangle \mathrm{d} s .
$$

As in Proposition 3.2 this follows from the (stochastic) Fubini theorem (see [14]). Therefore, the above estimates imply (4.1).

For Hilbert spaces $E$ we can prove a maximal regularity result in the nonautonomous setting. The autonomous case has been considered in [16, Theorem 6.14]. Our proof below is different from [16] even in the autonomous case. We briefly recalled some results on $H^{\infty}$-calculus in Section 2.2. Note that we use formulations for $A(t)$ instead of $-A(t)$.

Assume (AT1) and the following condition on the operators $(A(t))_{t \in[0, T]}$. 
$\left(H^{\infty}\right)$ There exists constant $w \in \mathbb{R}, C>0$ and $\left.\sigma \in \pi / 2, \pi\right)$ such that for all $t \in[0, T], A_{w}(t)$ has a bounded $H^{\infty}\left(\Sigma_{\sigma}\right)$-calculus on $\Sigma_{\varphi}$ and

$$
C:=\sup _{t \in[0, T]}\left(\left\{\left\|f\left(A_{w}(t)\right)\right\|:\|f\|_{H^{\infty}\left(\Sigma_{\sigma}\right)} \leq 1\right\}\right)<\infty .
$$

Condition $\left(H^{\infty}\right)$ has also appeared in [56] (with $A(t)$ replaced by $-A(t)$ ). In the autonomous $\left(H^{\infty}\right)$ has been used in [19] to obtain maximal regularity for equations with additive noise in Banach spaces. This has been extended to the non-autonomous setting in [56].

We reformulate the sufficient conditions from Remark 2.6 in our situation here.

REMARK 4.2. Each of the following two conditions is sufficient for $\left(H^{\infty}\right)$ for a Hilbert space $E$.

(1) If (AT1) holds and there exists a $w \in \mathbb{R}$, such that each $A_{w}(t)$ generates an analytic contraction semigroup, then $\left(H^{\infty}\right)$ holds.

(2) If there exists a $w \in \mathbb{R}$ such that each $-A_{w}(t)$ is positive and self-adjoint, then for all $\sigma \in(\pi / 2, \pi)$, the condition $\left(H^{\infty}\right)$ holds with $C=1$.

In the following result we use $\left(H^{\infty}\right)$ to obtain a maximal regularity result for the stochastic convolution. Recall that by $(2.5),\left\|\left(-A_{w}(t)\right)^{\frac{1}{2}} P(t, s)\right\| \leq C(t-s)^{-\frac{1}{2}}$.

THEOREM 4.3. Let $E$ be a Hilbert space. Assume that $(A(t))_{t \in[0, T]}$ satisfies (AT) and $\left(H^{\infty}\right)$. If $\Phi:[0, T] \times \Omega \rightarrow \gamma(H, E)$ is $H$-strongly measurable and adapted, then

$$
\mathbb{E}\left\|t \mapsto\left(-A_{w}(t)\right)^{\frac{1}{2}}(P \diamond \Phi)(t)\right\|_{L^{2}(0, T ; E)}^{2} \leq C \mathbb{E}\|\Phi\|_{L^{2}(0, T ; \gamma(H, E))}^{2},
$$

where $C$ is a constant independent of $\Phi$.

For second order partial differential equations the inequality (4.4) will allow us to derive $W^{1,2}(S)$-regularity, where $W^{1,2}(S)$ denotes the Sobolev space on a domain $S \subset \mathbb{R}^{n}$. Furthermore, (4.4) can be useful for non-linear equations.

Proof. First assume that $\Phi \in L^{2}\left(\Omega ; L^{2}(0, T ; \gamma(H, E))\right)$. Notice that $\gamma(H, E)=$ $\mathcal{C}_{2}(H, E)$ is the space of Hilbert-Schmidt operators from $H$ into $E$. Let $\left(h_{n}\right)_{n \geq 1}$ be an orthonormal basis for $H$. By the Itô isometry and the Fubini theorem, we have

$$
\begin{aligned}
\mathbb{E} \| t & \mapsto\left(-A_{w}(t)\right)^{\frac{1}{2}}(P \diamond \Phi)(t) \|_{L^{2}(0, T ; E)}^{2} \\
& =\mathbb{E} \int_{0}^{T} \int_{0}^{t}\left\|\left(-A_{w}(t)\right)^{\frac{1}{2}} P(t, s) \Phi(s)\right\|_{\gamma(H, E)}^{2} \mathrm{~d} s \mathrm{~d} t \\
& =\mathbb{E} \int_{0}^{T} \sum_{n \geq 1} \int_{s}^{T}\left\|\left(-A_{w}(t)\right)^{\frac{1}{2}} P(t, s) \Phi(s) h_{n}\right\|^{2} \mathrm{~d} t \mathrm{~d} s .
\end{aligned}
$$

Let $P_{w}(t, s)=\mathrm{e}^{w(t-s)} P(t, s)$. For $x \in E$ we can estimate

$$
\left(\int_{s}^{T}\left\|\left(-A_{w}(t)\right)^{\frac{1}{2}} P_{w}(t, s) x\right\|^{2} \mathrm{~d} t\right)^{\frac{1}{2}} \leq \sum_{i=1}^{3} R_{i} .
$$


Here

$$
R_{1}^{2}=\int_{s}^{T}\left\|\left(-A_{w}(t)\right)^{\frac{1}{2}} Z(t, s) x\right\|^{2} \mathrm{~d} t
$$

with $Z(t, s)=P_{w}(t, s)-\exp \left((t-s) A_{w}(t)\right)$. It follows from [57, p. 144] and [6, Lemma 3.2.1 and Theorem 3.2.2] that

$$
\left\|\left(-A_{w}(t)\right)^{\frac{1}{2}} Z(t, s)\right\| \leq C_{4}(t-s)^{-\frac{1}{2}+\frac{\kappa \mu, v}{2}} .
$$

Therefore, $R_{1}^{2} \lesssim T^{\kappa_{\mu, v}}\|x\|$. Second, by [57, (2.4)]

$$
\begin{aligned}
R_{2}^{2} & =\int_{s}^{T}\left\|\left(-A_{w}(t)\right)^{\frac{1}{2}} \exp \left((t-s) A_{w}(t)\right) x-\left(-A_{w}(s)\right)^{\frac{1}{2}} \exp \left((t-s) A_{w}(s)\right) x\right\|^{2} \mathrm{~d} t \\
& \lesssim \int_{s}^{T}(t-s)^{2 \kappa_{\mu, \nu}-1} \mathrm{~d} t\|x\| \lesssim T^{2 \kappa_{\mu, v}}\|x\| .
\end{aligned}
$$

Finally, by $\left(H^{\infty}\right)$ and $(2.10)$

$$
R_{3}^{2}=\int_{s}^{T}\left\|\left(-A_{w}(s)\right)^{\frac{1}{2}} \exp \left((t-s) A_{w}(s)\right) x\right\|^{2} \mathrm{~d} t \lesssim\|x\| .
$$

It follows that

$$
\left(\int_{S}^{T}\left\|\left(-A_{w}(t)\right)^{\frac{1}{2}} P(t, s) x\right\|^{2} \mathrm{~d} t\right)^{\frac{1}{2}} \lesssim\|x\| .
$$

We may conclude that

$$
\mathbb{E}\left\|\left(-A_{w}(\cdot)\right)^{\frac{1}{2}} P \diamond \Phi\right\|_{L^{2}(0, T ; E)}^{2} \lesssim \mathbb{E} \int_{0}^{T} \sum_{n \geq 1}\left\|\Phi(s) h_{n}\right\|^{2} \mathrm{~d} s=\mathbb{E}\|\Phi\|_{L^{2}(0, T ; \gamma(H, E))}^{2}
$$

This proves (4.4).

\section{The abstract evolution equation and solution concepts}

In this section and Section 6 let $E, H,(\Omega, \mathcal{F}, \mathbb{P}),\left(\mathcal{F}_{t}\right)_{t \in[0, T]}$, and $W_{H}$ be as in Section 4. On the Banach space $E$ we consider the problem

$$
\left\{\begin{aligned}
\mathrm{d} U(t) & =(A(t) U(t)+F(t, U(t))) \mathrm{d} t+B(t, U(t)) \mathrm{d} W_{H}(t), \quad t \in[0, T], \\
U(0) & =u_{0} .
\end{aligned}\right.
$$

Here, $(A(t))_{t \in[0, T]}$ is a family of closed unbounded operators on $E$. The initial value is a strongly $\mathcal{F}_{0}$-measurable mapping $u_{0}: \Omega \rightarrow E$.

We assume (AT) and (H1) $\eta_{0}$. We assume $a \in\left[0, \eta_{0}\right)$ and for each $(t, \omega) \in[0, T] \times \Omega$, we assume that $F$ and $B$ map as follows:

$$
\begin{aligned}
x & \mapsto F(t, \omega, x) \text { maps from } \widetilde{E}_{a}^{t} \text { into } E_{-1}^{t}, \\
x & \mapsto B(t, \omega, x) \text { maps from } \widetilde{E}_{a}^{t} \text { into } \gamma\left(H, E_{-1}^{t}\right) .
\end{aligned}
$$

More precisely, we have the following hypothesis on $F$ and $B$. 
(H2) Let $a \in\left[0, \eta_{0}\right)$ and $\theta_{F} \in[0, \mu)$ be such that $a+\theta_{F}<1$. For all $x \in \widetilde{E}_{a}$, $(t, \omega) \mapsto\left(-A_{w}(t)\right)^{-\theta_{F}} F(t, \omega, x) \in E$ is strongly measurable and adapted. The function $\left(-A_{w}(t)\right)^{-\theta_{F}} F$ has linear growth and is Lipschitz continuous in space uniformly in $[0, T] \times \Omega$, that is there are constants $L_{F}$ and $C_{F}$ such that for all $t \in[0, T], \omega \in \Omega, x, y \in \widetilde{E}_{a}$,

$$
\begin{aligned}
& \|\left(-A_{w}(t)\right)^{-\theta_{F}}(F(t, \omega, x)-F(t, \omega, y))\left\|_{E} \leq L_{F}\right\| x-y \|_{\widetilde{E}_{a}}, \\
&\left\|\left(-A_{w}(t)\right)^{-\theta_{F}} F(t, \omega, x)\right\|_{E} \leq C_{F}\left(1+\|x\|_{\widetilde{E}_{a}}\right) .
\end{aligned}
$$

(H3) Let $a \in\left[0, \eta_{0}\right)$ and $\theta_{B} \in[0, \mu)$ be such that $a+\theta_{B}<\frac{1}{2}$. For all $x \in \widetilde{E}_{a},(t, \omega) \mapsto$ $\left(-A_{w}(t)\right)^{-\theta_{B}} B(t, \omega, x) \in \gamma(H, E)$ is strongly measurable and adapted. The function $\left(-A_{w}\right)^{-\theta_{B}} B$ has linear growth and is Lipschitz continuous in space uniformly in $[0, T] \times \Omega$, that is there are constants $L_{B}$ and $C_{B}$ such that for all $t \in[0, T], \omega \in \Omega, x, y \in \widetilde{E}_{a}$,

$$
\begin{aligned}
\left\|\left(-A_{w}(t)\right)^{-\theta_{B}}(B(t, \omega, x)-B(t, \omega, y))\right\|_{\gamma(H, E)} & \leq L_{B}\|x-y\|_{\widetilde{E}_{a}}, \\
\left\|\left(-A_{w}(t)\right)^{-\theta_{B}} B(t, \omega, x)\right\|_{\gamma(H, E)} & \leq C_{B}\left(1+\|x\|_{\widetilde{E}_{a}}\right) .
\end{aligned}
$$

In our application in Section 8 we will not use functions $F$ which take values in extrapolation spaces. However, in forthcoming papers this will be important. In Section 7 we will consider locally Lipschitz coefficients $F$ and $B$.

We introduce variational and mild solutions for (SE) and give conditions under which both concepts are equivalent.

We need the adjoint operators $A(t)^{*}$. Note that these also satisfy (AT1). Since in our setting $E$ will be a UMD space with type 2, it will also be reflexive. Therefore, Kato's result implies that also $A(t)^{*}$ is densely defined (cf. [59, Section VIII.4]).

For $t \in[0, T]$ let

$$
\begin{aligned}
\Gamma_{t}=\left\{\varphi \in C^{1}\left([0, t] ; E^{*}\right):\right. & \text { for all } s \in[0, t] \varphi(s) \in D\left(A(s)^{*}\right) \\
& \text { and } \left.s \mapsto A(s)^{*} \varphi(s) \in C\left([0, t] ; E^{*}\right)\right\} .
\end{aligned}
$$

Fix some $t \in[0, T]$ and $\varphi \in \Gamma_{t}$. Formally, applying the Itô formula to $\langle U(t), \varphi(t)\rangle$ yields

$$
\begin{aligned}
& \langle U(t), \varphi(t)\rangle-\left\langle u_{0}, \varphi(0)\right\rangle \\
& =\int_{0}^{t}\left\langle U(s), \varphi^{\prime}(s)\right\rangle \mathrm{d} s+\int_{0}^{t}\left\langle U(s), A(s)^{*} \varphi(s)\right\rangle+\langle F(s, U(s)), \varphi(s)\rangle \mathrm{d} s \\
& \quad+\int_{0}^{t} B(s, U(s))^{*} \varphi(s) \mathrm{d} W_{H}(s) .
\end{aligned}
$$

DEFINITION 5.1. Assume (AT), (H1) $\eta_{0}$, (H2) and (H3). An $\widetilde{E}_{a}$-valued process $(U(t))_{t \in[0, T]}$ is called a variational solution of $(\mathrm{SE})$, if

(i) $U$ is strongly measurable and adapted, and in $L^{2}\left(0, T ; \widetilde{E}_{a}\right)$ a.s. 
(ii) for all $t \in[0, T]$ and all $\varphi \in \Gamma_{t}$, almost surely, (5.5) holds.

The integrand $B(s, U(s))^{*} \varphi(s)$ of the stochastic integral in (5.5) should be read as

$$
\left(\left(-A_{w}(s)\right)^{-\theta_{B}} B(s, U(s))\right)^{*}\left(-A_{w}(s)^{*}\right)^{\theta_{B}} \varphi(s) .
$$

It follows from (H3) that $s \mapsto\left(\left(-A_{w}(s)\right)^{-\theta_{B}} B(s, U(s))\right)^{*}$ is strongly measurable and adapted and in $L^{2}\left(0, T ; \mathcal{B}\left(E^{*}, H^{*}\right)\right)$ a.s. Moreover,

$$
s \mapsto\left(-A_{w}(s)^{*}\right)^{\theta_{B}} \varphi(s)=\left(-A_{w}(s)^{*}\right)^{-1+\theta_{B}}\left(-A_{w}(s)^{*}\right) \varphi(s)
$$

is in $C\left([0, t] ; E^{*}\right)$ by the Hölder continuity of $\left(-A_{w}(s)\right)^{-1+\theta_{B}}$ (cf. [48, (2.10) and (2.11)]) and its adjoint and the assumption on $\varphi$. The integrand $\langle F(s, U(s)), \varphi(s)\rangle$ has to be interpreted in a similar way.

Next we define a mild solution.

DEFINITION 5.2. Assume (AT), (H1) $\eta_{0},(\mathrm{H} 2)$ and (H3). Let $r \in(2, \infty)$ be such that $\theta_{F}<1-\frac{1}{r}$ and $\theta_{B}<\frac{1}{2}-\frac{1}{r}$. We call an $\widetilde{E}_{a}$-valued process $(U(t))_{t \in[0, T]}$ a mild solution of (SE), if

(i) $U$ is strongly measurable and adapted, and in $L^{r}\left(0, T ; \widetilde{E}_{a}\right)$ a.s.

(ii) for all $t \in[0, T]$, a.s.

$$
U(t)=P(t, 0) u_{0}+P * F(\cdot, U)(t)+P \diamond B(\cdot, U)(t) \text { in } E .
$$

Recall that $P * F(\cdot, U)$ and $P \diamond B(\cdot, U)$ stand for the convolution and stochastic convolution as defined in Sections 3 and 4, respectively.

The stochastic convolution is well defined. This follows if we write

$$
P(t, s) B(s, U(s))=P(t, s)\left(-A_{w}(s)\right)^{\theta_{B}}\left(-A_{w}(s)\right)^{-\theta_{B}} B(s, U(s))
$$

and therefore by (2.5) and Hölder's inequality

$$
\begin{aligned}
\int_{0}^{t}\|P(t, s) B(s, U(s))\|^{2} \mathrm{~d} s & \lesssim \int_{0}^{t}(t-s)^{-2 \theta_{B}}\left\|\left(-A_{w}(s)\right)^{-\theta_{B}} B(s, U(s))\right\|^{2} \mathrm{~d} s \\
& \lesssim 1+\|U\|_{L^{r}\left(0, T ; \widetilde{E}_{a}\right)}^{2} .
\end{aligned}
$$

In the same way one can see that the deterministic convolution is well defined. If $\theta_{F}=\theta_{B}=0$, then one may also take $r=2$ in Definition 5.2.

To prove equivalences between variational and mild solutions, we need the following condition:

(W) Assume that for all $t \in[0, T]$, there is a $\sigma\left(E^{*}, E\right)$-sequentially dense subspace $\Upsilon_{t}$ of $E^{*}$ such that for all $x^{*} \in \Upsilon_{t}$, we have $\varphi(s):=P(t, s)^{*} x^{*}$ is in $C^{1}\left([0, t] ; E^{*}\right)$ and $\varphi(s) \in D\left(A(s)^{*}\right)$ for all $s \in[0, t]$ and

$$
\frac{d}{\mathrm{~d} s} \varphi(s)=-A(s)^{*} \varphi(s) .
$$


The condition (W) was introduced in [56] in order to relate different solution concepts in the case of (SE) with additive noise.

REMARK 5.3. If (AT) holds for both for $A(t)$ and its adjoint, then (W) is fulfilled with $\Upsilon_{t}=D\left(\left(A(t)^{*}\right)^{2}\right)$. This follows from [2, Theorem 6.1]) and [3, p. 1176]. If $E$ is reflexive, by Kato's result [23], one may take $\Upsilon_{t}=D\left(A(t)^{*}\right)$.

PROPOSITION 5.4. Assume (AT), (H1) $\eta_{0},(\mathrm{H} 2),(\mathrm{H} 3)$ and $(\mathrm{W})$. Let $r \in(2, \infty)$ be such that $\theta_{B}<\frac{1}{2}-\frac{1}{r}$ and $\theta_{F}<1-\frac{1}{r}$. Let $U:[0, T] \times \Omega \rightarrow \widetilde{E}_{a}$ be strongly measurable and adapted and such that $U \in L^{r}\left(0, T ; \widetilde{E}_{a}\right)$ a.s. The following assertions are equivalent:

(1) E is a mild solution of (SE).

(2) $U$ is a variational solution of (SE).

Condition (W) is only needed in (2) $\Rightarrow(1)$. If $\theta_{F}=\theta_{B}=0$, then one may also take $r=2$ in Proposition 5.4. For the proof of the above equivalence we refer to the appendix.

\section{Existence, uniqueness, and regularity}

Assume (AT) and (H1) $\eta_{0}$. For $a \in\left[0, \eta_{0}\right)$ and $r \in[1, \infty)$ let $Z_{a}^{r}$ be the closed subspace of adapted processes in $C\left([0, T] ; L^{r}\left(\Omega ; \widetilde{E}_{a}\right)\right)$. Assume (H2) and (H3), where $a \in\left[0, \eta_{0}\right)$.

Define the fixed point operator $L: Z_{a}^{r} \rightarrow Z_{a}^{r}$ as

$$
L(\phi)=t \mapsto P(t, 0) u_{0}+P * F(\cdot, \phi)(t)+P \diamond B(\cdot, \phi)(t) .
$$

In the next lemma we show that $L$ is well-defined and that it is a strict contraction in $Z_{a}^{r}$ for a suitable equivalent norm. Recall that $P * F(\cdot, \phi)$ and $P \diamond B(\cdot, \phi)$ stand for the convolution and stochastic convolution as defined in Sections 3 and 4, respectively.

LEMMA 6.1. Assume (AT), (H1) $\eta_{0},(\mathrm{H} 2)$ and (H3). Let $r \in(2, \infty)$ be such that $a+\frac{1}{r}<\min \left\{\frac{1}{2}-\theta_{B}, 1-\theta_{F}, \eta_{0}\right\}$ and let $u_{0} \in L^{r}\left(\Omega, \mathcal{F}_{0} ; E_{a}^{0}\right)$. Then the operator $L$ is well-defined and there is an equivalent norm $\|\cdot\|$ on $Z_{a}^{r}$ such that for all $\phi_{1}, \phi_{2} \in Z_{a}^{r}$,

$$
\left\|L\left(\phi_{1}\right)-L\left(\phi_{2}\right)\right\| Z_{a}^{r} \leq \frac{1}{2}\left\|\phi_{1}-\phi_{2}\right\|_{Z_{a}^{r}}
$$

Moreover, there is a constant $C$ independent of $u_{0}$ such that for all $\phi \in Z_{a}^{r}$,

$$
\|L(\phi)\| Z_{a}^{r} \leq C\left(1+\left(\mathbb{E}\left\|u_{0}\right\|_{E_{a}^{0}}^{r}\right)^{\frac{1}{r}}\right)+\frac{1}{2}\|\phi\| Z_{Z_{a}^{r}} .
$$

Proof. Initial value part

By (2.4) we may estimate

$$
\left\|P(t, 0) u_{0}\right\|_{E_{a}^{t}} \leq C\left\|u_{0}\right\|_{E_{a}^{0}} .
$$


This clearly implies

$$
\left\|t \mapsto P(t, 0) u_{0}\right\|_{Z_{a}^{r}} \lesssim\left\|u_{0}\right\|_{L^{r}\left(\Omega ; E_{a}^{0}\right)}
$$

where the path continuity of $P(t, 0) u_{0}$ in $\widetilde{E}_{a}$ follows from Lemma 2.3.

Deterministic convolution

(a) Let $\left(-A_{w}\right)^{-\theta_{F}} \phi \in L^{\infty}\left(0, T ; L^{r}(\Omega ; E)\right)$. Recall from the proof of Proposition 3.2 that $P * \phi=\zeta=R_{\alpha}\left(\zeta_{\alpha}\right)$. It follows from (2.4) that for all $t \in[0, T]$,

$$
\begin{aligned}
\|P * \phi(t)\|_{L^{r}\left(\Omega ; \widetilde{E}_{a}\right)} & =\left\|R_{\alpha}\left(\zeta_{\alpha}\right)(t)\right\|_{L^{r}\left(\Omega ; \widetilde{E}_{a}\right)} \\
& \lesssim \int_{0}^{t}(t-s)^{\alpha-1-a}\left\|\zeta_{\alpha}(s)\right\|_{L^{r}(\Omega ; E)} \mathrm{d} s
\end{aligned}
$$

By (2.5) we obtain that

$$
\begin{aligned}
\left\|\zeta_{\alpha}(s)\right\|_{L^{r}(\Omega ; E)} & \lesssim\left\|\int_{0}^{s}(s-u)^{-\alpha}\right\| P(s, u) \phi(u)\|\mathrm{d} u\|_{L^{r}(\Omega)} \\
& \lesssim\left\|\int_{0}^{s}(s-u)^{-\alpha-\theta_{F}}\right\|\left(-A_{w}(u)\right)^{-\theta_{F}} \phi(u)\|\mathrm{d} u\|_{L^{r}(\Omega)} \\
& \leq \int_{0}^{s}(s-u)^{-\alpha-\theta_{F}}\left\|\left(-A_{w}(u)\right)^{-\theta_{F}} \phi(u)\right\|_{L^{r}(\Omega ; E)} \mathrm{d} u .
\end{aligned}
$$

If we combine this with (6.4) we obtain that for all $t \in[0, T]$

$$
\begin{aligned}
\| P & \diamond \phi(t) \|_{L^{r}\left(\Omega ; \widetilde{E}_{a}\right)} \\
& \lesssim \int_{0}^{t}(t-s)^{\alpha-1-a} \int_{0}^{s}(s-u)^{-\alpha-\theta_{F}}\left\|\left(-A_{w}(u)\right)^{-\theta_{F}} \phi(u)\right\|_{L^{r}(\Omega ; E)} \mathrm{d} u \mathrm{~d} s \\
& \approx \int_{0}^{t}(t-u)^{-a-\theta_{F}}\left\|\left(-A_{w}(u)\right)^{-\theta_{F}} \phi(u)\right\|_{L^{r}(\Omega ; E)} \mathrm{d} u,
\end{aligned}
$$

where in the last step we used Fubini's theorem and $\int_{0}^{1} s^{-\alpha-\theta_{F}}(1-s)^{\alpha-1-a} \mathrm{~d} s$ is finite. Note that $P \diamond \phi \in Z_{a}^{r}$ follows from the fact that $P \diamond \phi$ is also (Hölder)continuous by Proposition 3.2.

(b) Let $\phi_{1}, \phi_{2} \in Z_{a}^{r}$. Then by (H2), $\left(-A_{w}\right)^{-\theta_{F}} F\left(\cdot, \phi_{1}\right)$ and $\left(-A_{w}\right)^{-\theta_{F}} F\left(\cdot, \phi_{2}\right)$ are adapted and in $L^{\infty}\left(0, T ; L^{r}(\Omega ; E)\right)$ and by (a), $P * F\left(\cdot, \phi_{1}\right)$ and $P * F\left(\cdot, \phi_{2}\right)$ define an element of $Z_{a}^{r}$ and

$$
\begin{array}{rl}
\| P & * F\left(\cdot, \phi_{1}\right)(t)-P * F\left(\cdot, \phi_{2}\right)(t) \|_{L^{r}\left(\Omega ; \widetilde{E}_{a}\right)} \\
& \lesssim \int_{0}^{t}(t-s)^{-a}\left\|\left(-A_{w}(s)\right)^{-\theta_{F}} F\left(s, \phi_{1}(s)\right)-\left(-A_{w}(s)\right)^{-\theta_{F}} F\left(s, \phi_{2}(s)\right)\right\|_{L^{r}(\Omega ; E)} \mathrm{d} s \\
& \leq L_{F} \int_{0}^{t}(t-s)^{-a-\theta_{F}}\left\|\phi_{1}(s)-\phi_{2}(s)\right\|_{L^{r}\left(\Omega ; \widetilde{E}_{a}\right)} \mathrm{d} s .
\end{array}
$$




\section{Stochastic convolution}

(a) Let $\left(-A_{w}\right)^{-\theta_{B}} \Phi \in L^{\infty}\left(0, T ; L^{r}(\Omega ; \gamma(H, E))\right)$ be adapted. Recall from the proof of Theorem 4.1 that $P \diamond \Phi=\zeta=R_{\alpha}\left(\zeta_{\alpha}\right)$. It follows from (2.4) that for all $t \in[0, T]$,

$$
\begin{aligned}
\|P \diamond \Phi(t)\|_{L^{r}\left(\Omega ; \widetilde{E}_{a}\right)} & =\left\|R_{\alpha}\left(\zeta_{\alpha}\right)(t)\right\|_{L^{r}\left(\Omega ; \widetilde{E}_{a}\right)} \\
& \lesssim \int_{0}^{t}(t-s)^{\alpha-1-a}\left\|\zeta_{\alpha}(s)\right\|_{L^{r}(\Omega ; E)} \mathrm{d} s .
\end{aligned}
$$

By Proposition 2.8 and (2.5) we obtain that

$$
\begin{aligned}
\left\|\zeta_{\alpha}(s)\right\|_{L^{r}(\Omega ; E)}^{2} & \lesssim\left\|\int_{0}^{s}(s-u)^{-2 \alpha}\right\| P(s, u) \Phi(u)\left\|_{\gamma(H, E)}^{2} \mathrm{~d} u\right\|_{L^{r / 2}(\Omega)} \\
& \lesssim\left\|\int_{0}^{s}(s-u)^{-2 \alpha-2 \theta_{B}}\right\|\left(-A_{w}(u)\right)^{-\theta_{B}} \Phi(u)\left\|_{\gamma(H, E)}^{2} \mathrm{~d} u\right\|_{L^{r / 2}(\Omega)} \\
& \leq \int_{0}^{s}(s-u)^{-2 \alpha-2 \theta_{B}}\left\|\left(-A_{w}(u)\right)^{-\theta_{B}} \Phi(u)\right\|_{L^{r}(\Omega ; \gamma(H, E))}^{2} \mathrm{~d} u .
\end{aligned}
$$

If we combine this with (6.7) we obtain that for all $t \in[0, T]$

$$
\begin{aligned}
\| P & \diamond \Phi(t) \|_{L^{r}\left(\Omega ; \widetilde{E}_{a}\right)} \\
& \lesssim \int_{0}^{t}(t-s)^{\alpha-1-a}\left(\int_{0}^{s}(s-u)^{-2 \alpha-2 \theta_{B}}\left\|\left(-A_{w}(u)\right)^{-\theta_{B}} \Phi(u)\right\|_{L^{r}(\Omega ; \gamma(H, E))}^{2} \mathrm{~d} u\right)^{\frac{1}{2}} \mathrm{~d} s .
\end{aligned}
$$

Note that $P \diamond \Phi \in Z_{a}^{r}$ follows from the fact that $P \diamond \Phi$ is also (Hölder)-continuous by Theorem 4.1 .

(b) Let $\phi_{1}, \phi_{2} \in Z_{a}^{r}$. Then $\left(-A_{w}\right)^{-\theta_{B}} B\left(\cdot, \phi_{1}\right)$ and $\left(-A_{w}\right)^{-\theta_{B}} B\left(\cdot, \phi_{2}\right)$ are adapted and in $L^{\infty}\left(0, T ; L^{r}(\Omega ; \gamma(H, E))\right)$. Denote

$$
\Delta\left(\phi_{1}, \phi_{2}\right)(u)=\left(-A_{w}(u)\right)^{-\theta_{B}}\left(B\left(u, \phi_{1}(u)\right)-B\left(u, \phi_{2}(u)\right)\right) .
$$

By (a) we obtain that $P \diamond B\left(\cdot, \phi_{1}\right)$ and $P \diamond B\left(\cdot, \phi_{2}\right)$ are in $Z_{a}^{r}$ and

$$
\begin{aligned}
\| P & \diamond B\left(\cdot, \phi_{1}\right)(t)-P \diamond B\left(\cdot, \phi_{2}\right)(t) \|_{L^{r}\left(\Omega ; \widetilde{E}_{a}\right)} \\
& \lesssim \int_{0}^{t}(t-s)^{\alpha-1-a}\left(\int_{0}^{s}(s-u)^{-2 \alpha-2 \theta_{B}}\left\|\Delta\left(\phi_{1}, \phi_{2}\right)(u)\right\|_{L^{r}(\Omega ; \gamma(H, E))}^{2} \mathrm{~d} u\right)^{\frac{1}{2}} \mathrm{~d} s \\
& \leq L_{B} \int_{0}^{t}(t-s)^{\alpha-1-a}\left(\int_{0}^{s}(s-u)^{-2 \alpha-2 \theta_{B}}\left\|\phi_{1}(u)-\phi_{2}(u)\right\|_{L^{r}\left(\Omega ; \widetilde{E}_{a}\right)}^{2} \mathrm{~d} u\right)^{\frac{1}{2}} \mathrm{~d} s .
\end{aligned}
$$

\section{Conclusions}

It follows from the above considerations that $L$ is well defined. For $p \geq 0$ define an equivalent norm on $Z_{a}^{r}$ by

$$
\|\phi\|_{Z_{a}^{r}}=\sup _{t \in[0, T]} \mathrm{e}^{-p t}\|\phi(t)\|_{L^{r}\left(\Omega ; \widetilde{E}_{a}\right)} .
$$


We obtain that for $\phi_{1}, \phi_{2} \in Z_{a}^{r}$, we have

$$
\left\|L\left(\phi_{1}\right)-L\left(\phi_{2}\right)\right\| Z_{a}^{r} \leq R_{1}+R_{2},
$$

where

$$
\begin{aligned}
& R_{1}=\left\|P \diamond B\left(\cdot, \phi_{1}\right)(t)-P \diamond B\left(\cdot, \phi_{2}\right)(t)\right\|_{L^{r}\left(\Omega ; \widetilde{E}_{a}\right)}, \\
& R_{2}=\left\|P * F\left(\cdot, \phi_{1}\right)(t)-P * F\left(\cdot, \phi_{2}\right)(t)\right\|_{L^{r}\left(\Omega ; \widetilde{E}_{a}\right)} .
\end{aligned}
$$

It follows from (6.6) that

$$
\begin{aligned}
R_{1} & \lesssim \sup _{t \in[0, T]} \mathrm{e}^{-p t} \int_{0}^{t}(t-s)^{-a}\left\|\phi_{1}-\phi_{2}\right\|_{L^{r}\left(\Omega ; \widetilde{E}_{a}\right)} \mathrm{d} s \\
& =\sup _{t \in[0, T]} \int_{0}^{t} \mathrm{e}^{-p(t-s)}(t-s)^{-a} \mathrm{e}^{-p s}\left\|\phi_{1}(s)-\phi_{2}(s)\right\|_{L^{r}\left(\Omega ; \widetilde{E}_{a}\right)} \mathrm{d} s \\
& \leq \int_{0}^{T} \mathrm{e}^{-p s} s^{-a-\theta_{F}} \mathrm{~d} s\left\|\phi_{1}-\phi_{2}\right\|_{Z_{a}^{r}}=f\left(p, T, a, \theta_{F}\right)\left\|\phi_{1}-\phi_{2}\right\|_{Z_{a}^{r}},
\end{aligned}
$$

where $f\left(p, T, a, \theta_{F}\right) \downarrow 0$ as $p \rightarrow \infty$. Similarly, by (6.9)

$$
\begin{aligned}
R_{2} & \lesssim \sup _{t \in[0, T]} \mathrm{e}^{-p t} \int_{0}^{t}(t-s)^{\alpha-1-a}\left(\int_{0}^{s}(s-u)^{-2 \alpha-2 \theta_{B}}\left\|\phi_{1}(u)-\phi_{2}(u)\right\|_{L^{r}\left(\Omega ; \widetilde{E}_{a}\right)}^{2} \mathrm{~d} u\right)^{\frac{1}{2}} \mathrm{~d} s \\
& \leq \int_{0}^{T} \mathrm{e}^{-p s} s^{\alpha-1-a} \mathrm{~d} s\left(\int_{0}^{T} \mathrm{e}^{-2 p u} u^{-2 \alpha-2 \theta_{B}} \mathrm{~d} u\right)^{\frac{1}{2}}\left\|\phi_{1}-\phi_{2}\right\|_{Z_{a}^{r}} \\
& =g\left(p, T, a, \alpha, \theta_{B}\right)\left\|\phi_{1}-\phi_{2}\right\|_{Z_{a}^{r}},
\end{aligned}
$$

where $g\left(p, T, a, \alpha, \theta_{B}\right) \downarrow 0$ as $p \rightarrow \infty$.

Taking $p$ large gives (6.1). Moreover, the estimate (6.2) follows from (6.1) and

$$
\|L(0)\| Z_{a}^{r} \leq C\left(1+\left\|u_{0}\right\|_{L^{r}\left(\Omega ; E_{a}^{0}\right)}\right) .
$$

We can now obtain a first existence, uniqueness, and regularity result for (SE).

PROPOSITION 6.2. Assume (AT1), (AT2), (H1) $\eta_{0},(\mathrm{H} 2)$ and (H3). Let $r \in(2, \infty)$ be such that $a+\frac{1}{r}<\min \left\{\frac{1}{2}-\theta_{B}, 1-\theta_{F}, \eta_{0}\right\}$ and let $u_{0} \in L^{r}\left(\Omega, \mathcal{F}_{0} ; E_{a}^{0}\right)$. Then the following assertions hold:

(1) There exists a unique mild solution $U \in Z_{a}^{r}$ of (SE). Moreover, there exists a constant $C \geq 0$ independent of $u_{0}$ such that

$$
\|U\|_{Z_{a}^{r}} \leq C\left(1+\left(\mathbb{E}\left\|u_{0}\right\|_{E_{a}^{0}}^{r}\right)^{\frac{1}{r}}\right) .
$$

(2) There exists a version of $U$ in $L^{r}\left(\Omega ; C\left([0, T] ; \widetilde{E}_{a}\right)\right)$. Furthermore, for every $\delta, \lambda>0$ such that $\delta+a+\lambda+\frac{1}{r}<\min \left\{\frac{1}{2}-\theta_{B}, 1-\theta_{F}, \eta_{0}\right\}$ there exists a 
version of $U$ such that $U-P(\cdot, 0) u_{0}$ in $L^{r}\left(\Omega ; C^{\lambda}\left([0, T] ; \widetilde{E}_{\delta+a}\right)\right)$ and there is a constant $C$ independent of $u_{0}$ such that

$$
\left(\mathbb{E}\left\|\left(U-P(\cdot, 0) u_{0}\right)\right\|_{C^{\lambda}\left([0, T] ; \widetilde{E}_{\delta+a}\right)}^{r}\right)^{\frac{1}{r}} \leq C\left(1+\left(\mathbb{E}\left\|u_{0}\right\|_{E_{a}^{0}}^{r}\right)^{\frac{1}{r}} .\right.
$$

If $u_{0} \in L^{r}\left(\Omega ; E_{\delta+a+\lambda}^{0}\right)$, then the same regularity as in (6.11) can be derived for the solution $U$. Indeed, by Lemma $2.3 P(\cdot, 0) u_{0} \in L^{r}\left(\Omega ; C^{\lambda}\left([0, T] ; \widetilde{E}_{\delta+a}\right)\right)$.

Proof. (1) It follows from Lemma 6.1 that there exists a unique fix point $U \in Z_{a}^{r}$ of $L$. It is clear from the definition of $L$ that $U$ is the unique mild solution in $Z_{a}^{r}$.

(2) By Proposition 3.2 we obtain that

$$
\mathbb{E}\|P * F(\cdot, U)\|_{C^{\lambda}\left([0, T] ; \widetilde{E}_{a+\delta}\right)}^{r} \lesssim \mathbb{E}\left\|\left(-A_{w}\right)^{-\theta_{F}} F(\cdot, U)\right\|_{L^{r}(0, T ; E)}^{r} \lesssim 1+\|U\|_{Z_{a}^{r} .}
$$

It follows from Theorem 4.1 that

$$
\begin{aligned}
& \mathbb{E}\|P \diamond B(\cdot, U)\|_{C^{\lambda}\left([0, T] ; \widetilde{E}_{a+\delta}\right)}^{r} \lesssim \mathbb{E}\left\|\left(-A_{w}\right)^{-\theta_{B}} B(\cdot, U(s))\right\|_{L^{r}(0, T ; \gamma(H, E))}^{r} \\
& \quad \lesssim 1+\|U\|_{Z_{a}^{r} .}
\end{aligned}
$$

Define $\tilde{U}:[0, T] \times \Omega \rightarrow \widetilde{E}_{a}$ as

$$
\tilde{U}(t)=P(t, 0) u_{0}+P * F(\cdot, U)(t)+P \diamond B(\cdot, U)(t)
$$

where we take the versions of the convolutions as above. Clearly, $\tilde{U}=U$ in $Z_{a}^{r}$ and therefore $\tilde{U}$ is the required mild solution. Moreover, there is a constant $C$ such that

$$
\left.\mathbb{E} \| \tilde{U}-P(\cdot, 0) u_{0}\right) \|_{C^{\lambda}\left([0, T] ; \widetilde{E}_{a+\delta}\right)}^{r} \leq C\left(1+\|\tilde{U}\|_{Z_{a}^{r}}\right)
$$

Now (6.11) follows from (6.10).

THEOREM 6.3. Assume (AT1), (AT2), (H1) $\eta_{0}$, (H2) and (H3). Let $u_{0}: \Omega \rightarrow E_{a}^{0}$ be strongly $\mathcal{F}_{0}$ measurable. Then the following assertions hold:

(1) There exists a unique mild solution $U$ of (SE) with paths in $C\left([0, T] ; \widetilde{E}_{a}\right)$ a.s.

(2) For every $\delta, \lambda>0$ with $\delta+a+\lambda<\min \left\{\frac{1}{2}-\theta_{B}, 1-\theta_{F}, \eta_{0}\right\}$ there exists $a$ version of $U$ such that $U-P(\cdot, 0) u_{0}$ in $C^{\lambda}\left([0, T] ; \widetilde{E}_{\delta+a}\right)$ a.s.

As given below in Proposition 6.2 if $u_{0} \in E_{\delta+a+\lambda}^{0}$ a.s, then $U$ has a version with paths in $C^{\lambda}\left([0, T] ; \widetilde{E}_{\delta+a}\right)$ for $\delta$ and $\lambda$ as in Theorem 6.3 (2).

For the proof we need the following lemma.

LEMMA 6.4. Under the conditions of Proposition 6.2 let $U$ and $V$ in the space $L^{r}\left(\Omega ; C\left([0, T] ; \widetilde{E}_{a}\right)\right)$ be the mild solutions of (SE) with initial values $u_{0}$ and $v_{0}$ in $L^{r}\left(\Omega, \mathcal{F}_{0} ; E_{a}^{0}\right)$. Then almost surely on the set $\left\{u_{0}=v_{0}\right\}$ we have $U \equiv V$. 
Proof. Let $\Gamma=\left\{u_{0}=v_{0}\right\}$. Since $\Gamma$ is $\mathcal{F}_{0}$-measurable, it follows from Lemma 6.1 that

$$
\begin{aligned}
\left\|U \mathbf{1}_{\Gamma}-V \mathbf{1}_{\Gamma}\right\| Z_{r}^{a} & =\left\|L(U) \mathbf{1}_{\Gamma}-L(V) \mathbf{1}_{\Gamma}\right\| Z_{r}^{a} \\
& =\left\|\left(L\left(U \mathbf{1}_{\Gamma}\right)-L\left(V \mathbf{1}_{\Gamma}\right)\right) \mathbf{1}_{\Gamma}\right\|_{Z_{r}^{a}} \\
& \leq \frac{1}{2}\left\|U \mathbf{1}_{\Gamma}-V \mathbf{1}_{\Gamma}\right\| Z_{r}^{a}
\end{aligned}
$$

hence $\left.U\right|_{[0, T] \times \Gamma}=\left.V\right|_{[0, T] \times \Gamma}$ in $Z_{a}^{r}$. The result now follows from the path continuity of $U$ and $V$.

Proof of Theorem 6.3. Let $r>2$ be such that $\delta+a+\lambda+\frac{1}{r}<\min \left\{\frac{1}{2}-\theta_{B}, 1-\theta_{F}, \eta_{0}\right\}$. Define $\left(u_{n}\right)_{n \geq 1}$ in $L^{r}\left(\Omega, \mathcal{F}_{0} ; E_{a}^{0}\right)$ as $u_{n}=\mathbf{1}_{\left\{\left\|u_{0}\right\| \leq n\right\}} u_{0}$. By Proposition 6.2, for each $n \geq 1$, there is a mild solution $U_{n} \in Z_{a}^{r}$ of (SE) with initial value $u_{n}$ and we may take the version of $U_{n}$ from Proposition 6.2 (2). Lemma 6.4 implies that for $1 \leq m \leq n$ almost surely on the set $\left\{\left\|u_{0}\right\| \leq m\right\}$, for all $t \in[0, T], U_{n}(t)=U_{m}(t)$. It follows that almost surely, for all $t \in[0, T], \lim _{n \rightarrow \infty} U_{n}(t)$ exists in $\widetilde{E}_{a}$. Define $U:[0, T] \times \Omega \rightarrow$ $\widetilde{E}_{a}$ as $U(t)=\lim _{n \rightarrow \infty} U_{n}(t)$ if this limit exists and 0 otherwise. Clearly, $U$ is strongly measurable and adapted. Moreover, almost surely on $\left\{\left\|u_{0}\right\| \leq n\right\}$, for all $t \in[0, T]$, $U(t)=U_{n}(t)$, and hence $U-P(\cdot, 0) u_{0}$ has the same regularity as $U_{n}-P(\cdot, 0) u_{n}$. It can be easily checked that $U$ is a mild solution of (SE) satisfying (2).

Uniqueness: Let $U^{1}, U^{2} \in C\left([0, T] ; \widetilde{E}_{a}\right)$ a.s. be mild solutions of (SE). For each $n \geq 1$ and $i=1,2$ define the stopping times $\nu_{n}^{i}$ as

$$
v_{n}^{i}=\inf \left\{t \in[0, T]:\left\|U^{i}(t)\right\|_{\widetilde{E}_{a}} \geq n\right\} .
$$

For each $n \geq 1$ let $\tau_{n}=v_{n}^{1} \wedge v_{n}^{2}$, and let $U_{n}^{1}=U^{1} \mathbf{1}_{\left[0, \tau_{n}\right]}$ and $U_{n}^{2}=U^{2} \mathbf{1}_{\left[0, \tau_{n}\right]}$. Then for all $n \geq 1, U_{n}^{1}$ and $U_{n}^{2}$ are in $L^{r}\left(\Omega ; L^{\infty}\left(0, T ; \widetilde{E}_{a}\right)\right)$ for all $r<\infty$ so in particular in $L^{\infty}\left(0, T ; L^{r}\left(\Omega ; \widetilde{E}_{a}\right)\right)$ for all $r<\infty$. One easily checks that

$$
U_{n}^{i}=\mathbf{1}_{\left[0, \tau_{n}\right]}\left(L\left(U_{n}^{i}\right)\right)^{\tau_{n}}, \quad i=1,2,
$$

where $L$ is the mapping introduced before Lemma 6.1 and

$$
\left(L\left(U_{n}^{i}\right)\right)^{\tau_{n}}(t):=\left(L\left(U_{n}^{i}\right)\right)\left(t \wedge \tau_{n}\right), \quad i=1,2 .
$$

One can check that Lemma 6.1 remains valid if $Z_{a}^{r}$ is replaced by $\widehat{Z}_{a}^{r}$ the space of all adapted processes in $L^{\infty}\left(0, T ; L^{r}\left(\Omega ; \widetilde{E}_{a}\right)\right)$. Therefore,

$$
\begin{aligned}
\left\|U_{n}^{1}-U_{n}^{2}\right\| \widehat{Z}_{a}^{r} & =\left\|\mathbf{1}_{\left[0, \tau_{n}\right]}\left(L\left(U_{n}^{1}\right)^{\tau_{n}}-L\left(U_{n}^{2}\right)^{\tau_{n}}\right)\right\|_{\widehat{Z}_{a}^{r}} \\
& \leq\left\|L\left(U_{n}^{1}\right)-L\left(U_{n}^{2}\right)\right\| \widehat{Z}_{a}^{r} \\
& \leq \frac{1}{2}\left\|U_{n}^{1}-U_{n}^{2}\right\| \widehat{Z}_{a}^{r} .
\end{aligned}
$$

This implies that $U_{n}^{1}=U_{n}^{2}$ in $\widehat{Z}_{a}^{r}$. Therefore, for all $t \leq \tau_{n}, U^{1}(t)=U^{2}(t)$ a.s. Letting $n$ tend to infinity yields that for all $t \in[0, T], \bar{U}^{1}(t)=U^{2}(t)$ a.s. and by path-continuity this implies that a.s. for all $t \in[0, T], U^{1}(t)=U^{2}(t)$. 


\section{Local mild solutions}

Next we extend the results to the case where $F$ and $B$ are locally Lipschitz. This is a standard procedure (cf. [9,37,51] and references therein), but we believe it is better to include it here for completeness. Assume (AT) and (H1) $\eta_{\eta_{0}}$.

$(\mathrm{H} 2)^{\prime}$ Let $a \in\left[0, \eta_{0}\right)$ and $\theta_{F} \in[0, \mu)$ be such that $a+\theta_{F}<1$. For all $x \in \widetilde{E}_{a}$, $(t, \omega) \mapsto\left(-A_{w}(t)\right)^{-\theta_{F}} F(t, \omega, x) \in E$ is strongly measurable and adapted. The function $\left(-A_{w}(t)\right)^{-\theta_{F}} F$ is locally Lipschitz continuous in space uniformly in $[0, T] \times \Omega$, that is for each $R>0$ there is a constant $L_{F, R}$ such that for all $t \in[0, T], \omega \in \Omega, x, y \in \widetilde{E}_{a}$ with $\|x\|_{\widetilde{E}_{a}},\|y\|_{\widetilde{E}_{a}} \leq R$,

$$
\left\|\left(-A_{w}(t)\right)^{-\theta_{F}}(F(t, \omega, x)-F(t, \omega, y))\right\|_{E} \leq L_{F, R}\|x-y\|_{\widetilde{E}_{a}} .
$$

(H3) Let $a \in\left[0, \eta_{0}\right)$ and $\theta_{B} \in[0, \mu)$ be such that $a+\theta_{B}<\frac{1}{2}$. For all $x \in \widetilde{E}_{a}$, $(t, \omega) \mapsto\left(-A_{w}(t)\right)^{-\theta_{B}} B(t, \omega, x) \in E$ is strongly measurable and adapted. The function $\left(-A_{w}\right)^{-\theta_{B}} B$ has linear growth and is locally Lipschitz continuous in space uniformly in $[0, T] \times \Omega$, that is for each $R>0$ there is a constant $L_{B, R}$ such that for all $t \in[0, T], \omega \in \Omega, x, y \in \widetilde{E}_{a}$ with $\|x\|_{\widetilde{E}_{a}},\|y\|_{\widetilde{E}_{a}} \leq R$,

$$
\left\|\left(-A_{w}(t)\right)^{-\theta_{B}}(B(t, \omega, x)-B(t, \omega, y))\right\|_{\gamma(H, E)} \leq L_{B, R}\|x-y\|_{\widetilde{E}_{a}},
$$

We recall the definition of an admissible process and a local mild solution. Let $T>0$ and let $\tau$ be a stopping time with values in $[0, T]$. For $t \in[0, T]$ let

$$
\begin{aligned}
\Omega_{t}(\tau) & =\{\omega \in \Omega: t<\tau(\omega)\}, \\
{[0, \tau) \times \Omega } & =\{(t, \omega) \in[0, T] \times \Omega: 0 \leq t<\tau(\omega)\} .
\end{aligned}
$$

A process $\zeta:[0, \tau) \times \Omega \rightarrow E\left(\right.$ or $\left.(\zeta(t))_{t \in[0, \tau)}\right)$ is called admissible if for all $t \in[0, T]$, $\Omega_{t}(\tau) \ni \omega \rightarrow \zeta(t, \omega)$ is $\mathcal{F}_{t}$-measurable and for almost all $\omega \in \Omega,[0, \tau(\omega)) \ni t \mapsto$ $\zeta(t, \omega)$ is continuous.

DEFINITION 7.1. Assume (AT), $(\mathrm{H} 1)_{\eta_{0}},(\mathrm{H} 2)^{\prime}$ and $(\mathrm{H} 3)^{\prime}$. We call an admissible $\widetilde{E}_{a}$-valued process $(U(t))_{t \in[0, \tau)}$ a local mild solution of $(\mathrm{SE})$, if $\tau \in(0, T]$, $\tau=\lim _{n \rightarrow \infty} \tau_{n}$, where

$$
\tau_{n}=\inf \left\{t \in[0, T]:\|U(t)\|_{\widetilde{E}_{a}} \geq n\right\}, \quad n \geq 1
$$

and such that for all $t \in[0, T]$ and all $n \geq 1$, the following condition holds: for all $t \in[0, T]$, a.s.

$$
\begin{aligned}
U\left(t \wedge \tau_{n}\right)= & P\left(t \wedge \tau_{n}, 0\right) u_{0}+\int_{0}^{t \wedge \tau_{n}} P\left(t \wedge \tau_{n}, s\right) F(s, U(s \wedge \tau)) \mathbf{1}_{\left[0, \tau_{n}\right]}(s) \mathrm{d} s \\
& +I_{\tau_{n}}(B(\cdot, U))\left(t \wedge \tau_{n}\right) .
\end{aligned}
$$

In (7.1) we take $\tau_{n}=T$ if the infimum is taken over the empty set. By (H2) ${ }^{\prime}$ and Proposition 3.2 the deterministic convolution is well defined and pathwise continuous. 
The process $I_{\tau_{n}}(B(\cdot, U))$ is defined by

$$
I_{\tau_{n}}(B(\cdot, U))(t)=\int_{0}^{t} P(t, s) B\left(s, U\left(s \wedge \tau_{n}\right)\right) \mathbf{1}_{\left[0, \tau_{n}\right]}(s) \mathrm{d} W_{H}(s) .
$$

This process is well defined and pathwise continuous by Theorem 4.1. Therefore, $I_{\tau_{n}}(B(\cdot, U))\left(t \wedge \tau_{n}\right)$ is well defined. The motivation for defining $I_{\tau_{n}}$ in this way is explained in the appendix of [10]. It is needed in order to avoid the use of the process

$$
s \mapsto P\left(t \wedge \tau_{n}, s\right) B\left(s, U\left(s \wedge \tau_{n}\right)\right) \mathbf{1}_{\left[0, \tau_{n}\right]}(s),
$$

which is not adapted, since $P\left(t \wedge \tau_{n}, s\right) x$ is not adapted for $x \in E \backslash\{0\}$. This problem seems to be overlooked in some of the existing literature, and the referee kindly communicated the problem and [10] to the author.

For $a \in\left[0, \eta_{0}\right)$ and $r \in[1, \infty)$ let $Z_{a, \text { adm }}(\tau)$ be the space of $\widetilde{E}_{a}$-valued admissible

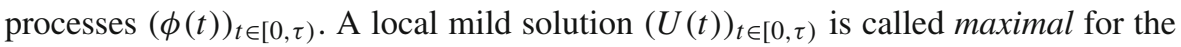
space $Z_{a, \text { adm }}(\tau)$ if for any other local mild solution $(\tilde{U}(t))_{t \in[0, \tilde{\tau})}$ in $Z_{a \text {, adm }}(\tau)$, almost surely we have $\tilde{\tau} \leq \tau$ and $\left.\tilde{U} \equiv U\right|_{[0, \tilde{\tau})}$. Clearly, a maximal local mild solution is always unique in $Z_{a \text {,adm }}(\tau)$. We say that a local mild solution $(U(t))_{t \in[0, \tau)}$ of (SE) is a global mild solution of (SE) if $\tau=T$ almost surely and $U$ has an extension to a mild solution $\hat{U}:[0, T] \times \Omega \rightarrow \widetilde{E}_{a}$ of (SE). In particular, almost surely "no blow" up occurs at $t=T$.

We say that $\tau$ is an explosion time if for almost all $\omega \in \Omega$ with $\tau(\omega)<T$,

$$
\limsup _{t \uparrow \tau(\omega)}\|U(t, \omega)\|_{\widetilde{E}_{a}}=\infty
$$

Notice that if $\tau=T$ almost surely, then $\tau$ is always an explosion time in this definition. However, there does not have to be any "blow up" in this case.

THEOREM 7.2. Assume (AT1), (AT2), (H1) $\eta_{0},(\mathrm{H} 2)^{\prime}$ and (H3)'. Let $u_{0}: \Omega \rightarrow E_{a}^{0}$ be strongly $\mathcal{F}_{0}$-measurable. Then the following assertions hold:

(1) There exists a unique maximal local mild solution $(U(t))_{[0, \tau)}$ in $Z_{a, a d m}(\tau)$ of (SE).

(2) For every $\lambda, \delta>0$ with $\lambda+\delta+a<\min \left\{1-\theta_{F}, \frac{1}{2}-\theta_{B}, \eta_{0}\right\}$ there exists $a$ version of $U$ such that for almost all $\omega \in \Omega$,

$$
t \mapsto U(t, \omega)-P(t, 0) u_{0}(\omega) \in C_{l o c}^{\lambda}\left([0, \tau(\omega)) ; \widetilde{E}_{a+\delta}\right) .
$$

If, additionally $F$ and $B$ are of linear growth, i.e., (5.2) and (5.4) hold, then the following assertions hold:

(3) The function $U$ from (1) and (2) is the unique global mild solution of (SE) with paths in $C\left([0, T] ; \widetilde{E}_{a}\right)$ and the statements of Theorem 6.3 hold.

(4) If $r \in(2, \infty)$ is such that $a+\frac{1}{r}<\min \left\{1-\theta_{F}, \frac{1}{2}-\theta_{B}, \eta_{0}\right\}$ and $u_{0} \in$ $L^{r}\left(\Omega, \mathcal{F}_{0} ; E_{a}^{0}\right)$, then the solution $U$ is in $Z_{a}^{r}$ and the statement of Proposition 6.2 hold. 
The proof is based on the following local uniqueness result.

LEMMA 7.3. Assume that the conditions of Theorem 7.2 are satisfied. Assume that $\left(U_{1}(t)\right)_{t \in\left[0, \tau_{1}\right)}$ in $Z_{a, a d m}\left(\tau_{1}\right)$ and $\left(U_{2}(t)\right)_{t \in\left[0, \tau_{2}\right)}$ in $Z_{a, a d m}\left(\tau_{2}\right)$ are local mild solutions of (SE) with initial values $u_{0}^{1}$ and $u_{0}^{2}$. Let $\Gamma=\left\{u_{0}^{1}=u_{0}^{2}\right\}$. Then almost surely on $\Gamma$, $\left.\left.U_{1}\right|_{\left[0, \tau_{1} \wedge \tau_{2}\right)} \equiv U_{2}\right|_{\left[0, \tau_{1} \wedge \tau_{2}\right)}$. Moreover, if $\tau_{1}$ is an explosion time for $U_{1}$, then almost surely on $\Gamma, \tau_{1} \geq \tau_{2}$. If $\tau_{1}$ and $\tau_{2}$ are explosion times for $U_{1}$ and $U_{2}$, then almost surely on $\Gamma, \tau_{1}=\tau_{2}$ and $U_{1} \equiv U_{2}$.

Both results can be proved using standard localization techniques. We refer the reader to [9, Section 4], [51, Section 5], [37, Section 8] or [55, Chapter 8] for a proof in a framework close to the one above.

\section{Examples}

Below, we consider the stochastic partial differential equation from [47]. We will apply Theorem 6.3 and Theorem 7.2 to obtain existence, uniqueness, and regularity of mild solutions. By Proposition 5.4 this will also give the unique variational solution. The operator $A(t)$ will be a time-dependent second-order elliptic differential operator with (time-dependent) Neumann boundary conditions. As in [47] we consider secondorder equations with noise that is white with respect to the time variable and colored with respect to space variable. We will reprove and improve some of the regularity results from [47] using the results of the previous sections. This will be done in three examples below.

Recall that $a$ is the parameter for the solution space $\widetilde{E}_{a}$. For the Examples 8.2 and 8.6 it will suffice to take $a=0$ in Theorem 6.3. In Example 8.9 we consider the locally Lipschitz case, and there we need $a>0$. The parameter $\theta_{B}$ allows us to consider covariance operators which are not necessarily of trace class. For details on covariance operators we refer to $[7,16]$.

REMARK 8.1. Some other examples which fit into our general framework are

(1) Higher order equations, possibly driven by multiplicative space-time white noise. Note that for second-order equations, this is only possible for dimension one, and therefore not very illustrative for our setting. In regular bounded domains in $\mathbb{R}^{n}$ one can consider multiplicative space-time white noise if the order of the elliptic operator $2 m>n$ (see [37] for the autonomous case and [55, Chapter 8] for the non-autonomous case with Dirichlet boundary conditions).

(2) $F$ and $B$ could be (non)-linear differential operators of lower order.

(3) Equations with boundary noise. This is work in progress [49].

Let us first recall some basic notations (cf. [54]). Let $S$ be a bounded domain and $m \in \mathbb{N}, p, q \in[1, \infty], s \in \mathbb{R}, \beta_{1}, \beta_{2} \in(0,1)$. $W^{m, p}(S)$ will be the Sobolev space. $B_{p, q}^{s}(S)$ will be the Besov space. $H^{s, p}(S)$ is the Bessel potential space and 
$H^{S}(S):=H^{2, p}(S)$, and $H^{m, p}(S)=W^{m, p}(S) . C^{\delta}(\bar{S})$ is the space of $\delta$-Hölder continuous functions. $C^{\beta_{1}, \beta_{2}}(\bar{S} \times[0, T])$ is the space of functions $f: S \times[0, T] \rightarrow \mathbb{R}$ which satisfy

$$
\begin{aligned}
& \left|f\left(s_{1}, t\right)-f\left(s_{2}, t\right)\right| \leq C_{1}\left|s_{1}-s_{2}\right|^{\beta_{1}}, s_{1}, s_{2} \in S, t \in[0, T], \\
& \left|f\left(s, t_{1}\right)-f\left(s, t_{2}\right)\right| \leq C_{2}\left|t_{1}-t_{2}\right|^{\beta_{2}}, s \in S, t_{1}, t_{2} \in[0, T]
\end{aligned}
$$

for certain constants $C_{1}, C_{2} \geq 0$. Clearly, $C^{\beta_{1}, \beta_{2}}(\bar{S} \times[0, T]) \hookrightarrow C^{\beta_{1} \wedge \beta_{2}}(\bar{S} \times[0, T])$.

EXAMPLE 8.2. Let $(\Omega, \mathcal{F}, \mathbb{P})$ be a complete probability space with a filtration $\left(\mathcal{F}_{t}\right)_{t \in[0, T]}$. Consider

$$
\begin{aligned}
\mathrm{d} u(t, s)= & A(t, s, D) u(t, s)+f(t, s, u(t, s)) \mathrm{d} t \\
& +g(t, s, u(t, s)) \mathrm{d} W(t, s), \quad t \in(0, T], \quad s \in S, \\
C(t, s, D) u(t, s)= & 0, \quad t \in(0, T], \quad s \in \partial S \\
u(0, s)= & u_{0}(s), \quad s \in S .
\end{aligned}
$$

Here $S$ is a bounded domain with boundary of class $C^{2}$ and outer normal vector $n(s)$ in $\mathbb{R}^{n}$, and

$$
\begin{aligned}
& A(t, s, D)=\sum_{i, j=1}^{n} D_{i}\left(a_{i j}(t, s) D_{j}\right)+a_{0}(t, s), \\
& C(t, s, D)=\sum_{i, j=1}^{n} a_{i j}(t, s) n_{i}(s) D_{j} .
\end{aligned}
$$

We assume that the coefficients are real and satisfy

$$
\begin{aligned}
a_{i j} & \in C^{\mu}([0, T] ; C(\bar{S})), a_{i j}(t, \cdot) \in C^{1}(\bar{S}), D_{k} a_{i j} \in C([0, T] \times \bar{S}), \\
a_{0} & \in C^{\mu}\left([0, T], L^{n}(S)\right) \cap C([0, T] ; C(\bar{S}))
\end{aligned}
$$

for $i, j, k=1, \ldots, n, t \in[0, T]$, and a constant $\mu \in\left(\frac{1}{2}, 1\right]$. Furthermore, let $\left(a_{i j}\right)$ be symmetric and assume that there exists a $\kappa>0$ such that

$$
\sum_{i, j=1}^{n} a_{i j}(t, s) \xi_{i} \xi_{j} \geq \kappa|\xi|^{2}, \quad s \in \bar{S}, \quad t \in[0, T], \quad \xi \in \mathbb{R}^{n} .
$$

Let $f, g:[0, T] \times \Omega \times S \times \mathbb{R} \rightarrow \mathbb{R}$ be measurable, adapted, and Lipschitz functions with linear growth uniformly $\Omega \times[0, T] \times S$, i.e., there exist $L_{f}, C_{f}, L_{g}, C_{g}$ such that for all $t \in[0, T], \omega \in \Omega, s \in \mathbb{R}$ and $x, y \in \mathbb{R}$,

$$
\begin{gathered}
|f(t, \omega, s, x)-f(t, \omega, s, y)| \leq L_{f}|x-y|, \\
|f(t, \omega, s, x)| \leq C_{f}(1+|x|), \\
|g(t, \omega, s, x)-g(t, \omega, s, y)| \leq L_{g}|x-y|, \\
|g(t, \omega, s, x)| \leq C_{g}(1+|x|) .
\end{gathered}
$$


The noise term $W$ is an $L^{2}(S)$-valued Brownian motion with respect to $\left(\mathcal{F}_{t}\right)_{t \in[0, T]}$. We assume that it has a covariance $Q \in \mathcal{B}\left(L^{2}(S)\right)$ which satisfies

$$
\sqrt{Q} \in \mathcal{B}\left(L^{2}(S), L^{\infty}(S)\right) .
$$

The following statements hold:

(1) Let $p \in[2, \infty)$. If $u_{0} \in L^{p}(S)$ a.s., then there exists a unique mild and variational solution $u$ of (8.1) with paths in $C\left([0, T] ; L^{p}(S)\right)$ a.s. Moreover, $u \in$ $L^{2}\left(0, T ; W^{1,2}(S)\right)$ a.s.

(2) If $u_{0} \in C^{1}(\bar{S})$ a.s., then the solution $u$ is in $C^{\lambda}\left([0, T] ; C^{2 \delta}(S)\right)$ for all $\lambda, \delta>0$ such that $\lambda+\delta<\frac{1}{2}$. In particular, $u \in C^{\beta_{1}, \beta_{2}}(\bar{S} \times[0, T])$ for all $\beta_{1} \in(0,1)$ and $\beta_{2} \in\left(0, \frac{1}{2}\right)$.

If in (1) $u_{0} \in L^{r}\left(\Omega ; L^{p}(S)\right)$ for some $r \in(2, \infty)$, then also

$$
\mathbb{E} \sup _{t \in[0, T]}\|u(t)\|_{L^{p}(S)}^{r} \lesssim \mathbb{E}\left\|u_{0}\right\|_{L^{p}(S)}^{r}
$$

This example improves [47, Theorem 3] in several ways:

REMARK 8.3. The assumptions on the coefficients $a_{i j}$ and the domain $S$ we have made are weaker than the ones in [47, p. 705]. The initial value in [47] is assumed to be more regular than ours (i.e. $u_{0} \in C^{2+\alpha}(\bar{S})$ instead of $C^{1}(\bar{S})$ ) and it has to fulfill the Neumann boundary condition at $t=0$. We consider $f$ and $g$ also depending on $[0, T] \times \Omega \times S$. In [47, Theorem 3] the obtained regularity is $C^{\beta_{1}, \beta_{2}}(\bar{S} \times[0, T])$ for all $\beta_{1} \in(0, \alpha)$ and $\beta_{2} \in\left(0, \frac{\alpha}{2} \wedge \frac{2}{n+2}\right)$. Here $\alpha \in(0,1)$ is a parameter which states how regular the coefficients $a_{i j}$ and the domain $S$ are. Even in the limiting case $\alpha=1$, our time regularity is better and it does not depend on the dimension $n$.

The condition on the noise term in [47] is formulated as (8.8) below.

REMARK 8.4. Since $Q$ is compact and positive, we can always find positive numbers $\left(\lambda_{n}\right)_{n \geq 1}$ and an orthonormal system $\left(e_{n}\right)_{n \geq 1}$ in $L^{2}(S)$ with $\sqrt{Q}=\sum_{n \geq 1} \lambda_{n} e_{n} \otimes$ $e_{n}$. It follows that we may decompose $W$ as

$$
W(t, s)=\sum_{n \geq 1} \sqrt{\lambda_{n}} W_{n}(t) e_{n}(s) .
$$

Here, $\left(W_{n}\right)_{n \geq 1}$ are independent real-valued standard Brownian motions.

The condition $\sqrt{Q} \in \mathcal{B}\left(L^{2}(S), L^{\infty}(S)\right)$ is for instance satisfied if $\left(e_{n}\right)_{n \geq 1}$ in $L^{\infty}(S)$ and

$$
\sum_{n \geq 1} \lambda_{n}\left\|e_{n}\right\|_{L^{\infty}(S)}^{2}<\infty .
$$

Indeed, for all $h \in L^{2}(S)$, by the Cauchy-Schwartz inequality

$$
|\sqrt{Q} h(s)|=\left|\sum_{n \geq 1} \sqrt{\lambda_{n}} e_{n}(s)\left[e_{n}, h\right]_{L^{2}(S)}\right| \leq\left(\sum_{n \geq 1} \lambda_{n}\left|e_{n}(s)\right|^{2}\right)^{\frac{1}{2}}\|h\|_{L^{2}(S)}<\infty
$$

for almost all $s \in S$. 
Proof of Example 8.2. Let $E=L^{p}(S)$ with $p \in[2, \infty)$. Then conditions (AT1) and (AT2) are satisfied (cf. $[1,48,58])$. Further, $(\mathrm{H} 1)_{\eta_{0}}$ is satisfied with $\eta_{0}=1$ and (cf. [54, Theorem 4.3.1.2])

$$
\widetilde{E}_{\eta}:=\left(L^{p}(S), W^{2, p}(S)\right)_{\eta, 2}=B_{p, 2}^{2 \eta}(S)
$$

for $\eta \in(0,1]$ and $\widetilde{E}_{0}=E$. Note that these spaces are all UMD spaces with type 2 as follows from the explanation after (2.12).

Let $F:[0, T] \times \Omega \times E \rightarrow E$ be defined by $F(t, \omega, x)(s)=f(t, \omega, s, x(s))$. Then, $F$ satisfies (H2). Let $B:[0, T] \times \Omega \times E \rightarrow \gamma\left(L^{2}(S), E\right)$ be defined as

$$
(B(t, \omega, x) h)(s)=b(t, \omega, s, x(s))(\sqrt{Q} h)(s) .
$$

This is well defined by the assumptions, and it satisfies (H3). Indeed, under condition (8.7), we obtain from Lemma 2.7 that for $x \in L^{p}(S)$,

$$
\|x \sqrt{Q}\|_{\gamma\left(L^{2}(S), L^{p}(S)\right)} \lesssim_{p} K\|x\|_{L^{p}(S)} .
$$

and therefore, for $x_{1}, x_{2} \in L^{p}(S)$,

$$
\begin{aligned}
\left\|B\left(t, \omega, x_{1}\right)-B\left(t, \omega, x_{2}\right)\right\|_{\gamma\left(L^{2}(S), L^{p}(S)\right)} & \lesssim_{p} K\left\|x_{1}-x_{2}\right\|_{L^{p}(S)}, t \in[0, T], \quad \omega \in \Omega, \\
\|B(t, \omega, x) h\|_{\gamma\left(L^{2}(S), L^{p}(S)\right)} & \leq K\left(1+\|x\|_{L^{p}(S)}\right), t \in[0, T], \quad \omega \in \Omega .
\end{aligned}
$$

By Theorem 6.3 with $a=\theta_{F}=\theta_{B}=0$, we obtain that there exists a unique mild solution $U$ with paths in $C([0, T] ; E)$ a.s.

Next, we use Proposition 5.4 to show that $U$ is also the unique variational solution in $C([0, T] ; E)$. Note that condition (W) is satisfied since $A(t)$ is self-adjoint in the sense that $A(t)^{*}$ on $L^{p}(S)$ is $A(t)$ on $L^{p^{\prime}}(S)$. Therefore, (AT2) holds for $A(t)^{*}$ and thus (W) holds by Remark 5.3. The result now follows from Proposition 5.4.

We still need to show that $U \in L^{2}\left(0, T ; H^{1}(S)\right)$ a.s. if $u_{0} \in L^{2}(S)$ a.s. Let $E=L^{2}(S)$. It follows from Remark 4.2 that $(A(t))_{t \in[0, T]}$ satisfies $\left(H^{\infty}\right)$. Since $A(t)$ is associated with a quadratic form with $V=W^{1,2}(S)$, it follows that $D\left(\left(-A_{w}(t)\right)^{\frac{1}{2}}\right)=W^{1,2}(S)$ for $w$ large enough with constants uniformly in time (cf. [52, Section 2.2]). We have already shown that $U \in C([0, T] ; E)$ a.s. Clearly, $B(U)$ is an element of $L^{\infty}\left([0, T] ; \gamma\left(L^{2}(S), E\right)\right)$ and by Theorem 4.3, $P \diamond B(U) \in$ $L^{2}\left(0, T ; W^{1,2}(S)\right)$ a.s. For the deterministic convolution, it follows from (3.2) that $P * F(U) \in L^{2}\left(0, T ; W^{1,2}(S)\right)$. Finally, by (4.5)

$$
\int_{0}^{T}\left\|P(t, 0) u_{0}\right\|_{W^{1,2}(S)}^{2} \mathrm{~d} s \lesssim \int_{0}^{T}\left\|\left(-A_{w}(t)\right)^{\frac{1}{2}} P(t, 0) u_{0}\right\|_{L^{2}(S)}^{2} \mathrm{~d} t \lesssim\left\|u_{0}\right\|_{L^{2}(S)}^{2} .
$$

This completes the proof.

(2) Let $E=L^{p}(S)$ for $p \in[2, \infty)$. If $u_{0} \in C^{1}(\bar{S})$ a.s., then we claim that $u_{0} \in E_{b}^{0}$ a.s. for all $b \in\left[0, \frac{1}{2}\right)$. Indeed, it suffices to show that $u_{0} \in[E, D(A(0))]_{\frac{1}{2}}$. By $[5$, Theorem 7.2 and Remark 7.3] and [18, Theorem 2.3] (also see Example 2.2), one has

$$
[E, D(A(0))]_{\frac{1}{2}}=\left[L^{p}(S), W^{2, p}(S)\right]_{\frac{1}{2}}=W^{1, p}(S) .
$$


Since $C^{1}(\bar{S}) \hookrightarrow W^{1, p}(S)$, the claim follows.

By Theorem 6.3 the process $U$ has the following regularity property: $U \in$ $C^{\lambda}\left([0, T] ; E_{\delta}\right)$ a.s. for all $\lambda, \delta>0$ such that $\lambda+\delta<\frac{1}{2}$. In particular, taking $p$ large it follows from [54, Theorem 4.6.1(e)] that $U \in C^{\lambda}\left([0, T] ; C^{2 \delta}(S)\right)$ for all $\lambda, \delta>0$ such that $\lambda+\delta<\frac{1}{2}$.

The final assertion follows from (6.11).

Let us show that the variational solution of Example 8.2 is also a variational solution of the second type as defined in [47].

REMARK 8.5. The variational solution of Example 8.7 satisfies: for all $t \in(0, T]$, $\varphi \in C^{1}\left([0, t] ; L^{2}(S)\right)$ such that $A(r, \cdot, D) \varphi \in C^{1}\left([0, t] ; L^{2}(S)\right)$, a.s.

$$
\begin{aligned}
\int_{S} u & (t, s) \varphi(t, s) \mathrm{d} s-\int_{S} u_{0}(s) \varphi(0, s) \mathrm{d} s \\
= & \int_{0}^{t} \int_{S} u(r, s) \varphi^{\prime}(r, s) \mathrm{d} s \mathrm{~d} r+\int_{0}^{t} \int_{S} u(r, s) A(r, s, D) \varphi(r, s) \mathrm{d} s \mathrm{~d} r \\
& +\int_{0}^{t} \int_{S} f(r, s, u(r, s)) \varphi(r, s) \mathrm{d} s \mathrm{~d} r \\
& +\sum_{n \geq 1} \int_{0}^{t} \int_{S} b(r, s, u(r, s)) e_{n}(s) \varphi(r, s) \mathrm{d} W_{n}(r)
\end{aligned}
$$

Therefore, by integration by parts and approximation it follows that for all $t \in(0, T]$, $\varphi \in W^{1,2}((0, t) \times S)$, a.s.

$$
\begin{aligned}
\int_{S} u(t, s) \varphi(t, s) \mathrm{d} s-\int_{S} u_{0}(s) \varphi(0, s) \mathrm{d} s \\
=\int_{0}^{t} \int_{S} u(r, s) \varphi^{\prime}(r, s) \mathrm{d} s \mathrm{~d} r-\int_{0}^{t} \int_{S}\langle\nabla u(r, s), a(r, s) \nabla \varphi(r, s)\rangle_{\mathbb{R}^{n} \mathrm{~d} s \mathrm{~d} r} \\
\quad+\int_{0}^{t} \int_{S} f(r, s, u(r, s)) \varphi(r, s) \mathrm{d} s \mathrm{~d} r \\
\quad+\sum_{n \geq 1} \int_{0}^{t} \int_{S} b(r, s, u(r, s)) \sqrt{Q} e_{n}(s) \varphi(r, s) \mathrm{d} W_{n}(r) .
\end{aligned}
$$

This coincides with the variational solution of the second kind from [47].

In the next example we will weaken the assumption on the covariance $Q$.

EXAMPLE 8.6. Consider equation (8.1) again. Assume the same conditions as in Example 8.2, but with (8.7) replaced by: there exist $\beta \in\left(0, \frac{1}{2}\right)$ and $q \in\left(\frac{n}{1-2 \beta}, \infty\right)$

$$
\sqrt{Q} \in \mathcal{B}\left(L^{2}(S), L^{q}(S)\right)
$$

The following statements hold: 
(1) Let $p \in[2, \infty)$ be such that $p>\left(n^{-1}-q^{-1}\right)^{-1}$. If $u_{0} \in L^{p}(S)$ a.s., then there exists a unique mild and variational solution $u$ of (8.1) with paths in $C\left([0, T] ; L^{p}(S)\right)$ a.s.

(2) If $u_{0} \in C^{1}(\bar{S})$ a.s., then the solution $u$ is in $C^{\lambda}\left([0, T] ; C^{2 \delta}(S)\right)$ for all $\lambda, \delta>0$ such that $\lambda+\delta<\beta$. In particular, $u \in C^{\beta_{1}, \beta_{2}}(\bar{S} \times[0, T])$ for all $\beta_{1} \in(0,2 \beta)$ and $\beta_{2} \in(0, \beta)$.

This example improves [47, Theorem 4] in similar ways as explained in Remark 8.3. Their condition on the noise term is formulated as (8.11) below.

REMARK 8.7. Assume that $Q$ is compact and has the same form as in Remark 8.4. The condition $\sqrt{Q} \in \mathcal{B}\left(L^{2}(S), L^{q}(S)\right)$ is for instance satisfied if $\left(e_{n}\right)_{n \geq 1}$ in $L^{q}(S)$ and

$$
\sum_{n \geq 1} \lambda_{n}\left\|e_{n}\right\|_{L^{q}(S)}^{2}<\infty .
$$

Indeed, without loss of generality we may assume that $q>2$. Taking the $L^{q}(S)$ norm on both sides in (8.9) yields

$$
\begin{aligned}
\|\sqrt{Q} h\|_{L^{q}(S)} & \leq\left\|\left(\sum_{n \geq 1} \lambda_{n}\left|e_{n}(s)\right|^{2}\right)^{\frac{1}{2}}\right\| h \|_{L^{2}(S)} \\
& \leq\left(\sum_{n \geq 1} \lambda_{n}\left\|e_{n}\right\|_{L^{q}(S)}^{2}\right)^{\frac{1}{2}}\|h\|_{L^{2}(S)}<\infty .
\end{aligned}
$$

REMARK 8.8. We should note that it is stated in [47, Theorem 4 with $\alpha=1$ ] that the space regularity of the solution becomes $C^{\sigma}(S)$ for all $\sigma<1$. We could not follow this argument. It seems that for the definition of $Y_{\delta}$ in [47, Lemma 4] one has restrictions on their parameter $\delta$ in terms of the $\beta$ from (8.10).

For example, consider the case that $S=(0,1), A=\frac{d^{2}}{d s^{2}}$ with Neumann boundary conditions, $f=0, b(x)=x$ and the noise is of the form $W(t, x)=e_{1}(x) W_{1}(t)$, where $e_{1} \in L^{q}(S)$ and $W_{1}$ is a standard Brownian motion. We do not believe that the solution has space regularity $C^{\sigma}(S)$ for all $\sigma<1$, in general.

Proof of Example 8.6. We proceed as in Example 8.2 but due to (8.11) we need to take $\theta_{B}>0$.

(1) Let $E=L^{p}(S)$. Since $Q \in \mathcal{B}\left(L^{2}(S)\right)$ we can assume that $q \geq 2$. Let $r \in(1, \infty)$ be such that $r\left(\frac{1}{p}+\frac{1}{q}\right)=1$. Let $\theta_{B} \in\left(\frac{n}{2 r}, \frac{1}{2}\right)$. This is possible by the restriction on $p$.

Let $w \in \mathbb{R}$ be so large that $\lambda \in \rho\left(A_{w}\right)$ for all $\operatorname{Re}(\lambda) \leq 0$. We claim that for $x \in L^{p}(S)$ and $h \in L^{2}(S)$,

$$
\left\|\left(-A_{w}(t)\right)^{-\theta_{B}} x \sqrt{Q} h\right\|_{L^{\infty}(S)} \lesssim\|x\|_{L^{p}(S)}\|h\|_{L^{2}(S)}
$$


with constants uniformly in $t \in[0, T]$. Indeed, fix $\theta_{B}^{\prime} \in\left(\frac{n}{2 r}, \theta_{B}\right)$. By [54, Theorem 4.6.1(e)] it follows that

$$
\|y\|_{L^{\infty}(S)} \lesssim\|y\|_{B_{r, 2}^{2 \theta_{B}^{\prime}}(S)}, \quad y \in B_{r, 2}^{2 \theta_{B}}(S) .
$$

Moreover,

$D\left(\left(-A_{w}(t)\right)_{B}^{\theta}\right) \hookrightarrow\left(L^{r}(S), D(A(t))\right)_{\theta_{B}^{\prime}, 2} \hookrightarrow\left(L^{r}(S), W^{2, r}(S)\right)_{\theta_{B}^{\prime}, 2}=B_{r, 2}^{2 \theta_{B}^{\prime}}(S)$

with embedding constants independent of $t \in[0, T]$. Here $D(A(t))$ stands for the domain of $A(t)$ in $L^{r}(S)$ and similarly for the fractional domain space. Therefore,

$$
\left\|A^{-\theta_{B}}(t) y\right\|_{L^{\infty}(S)} \lesssim\|y\|_{L^{r}(S)}, \quad y \in L^{r}(S)
$$

From this and Hölder's inequality we obtain that

$$
\begin{aligned}
\left\|\left(-A_{w}(t)\right)^{-\theta_{B}} x \sqrt{Q} h\right\|_{L^{\infty}(S)} & \lesssim\|x \sqrt{Q} h\|_{L^{r}(S)} \\
& \leq\|\sqrt{Q}\|_{\mathcal{B}\left(L^{2}(S), L^{q}(S)\right)}\|x\|_{L^{p}(S)}\|h\|_{L^{2}(S)} .
\end{aligned}
$$

The claim and Lemma 2.7 imply that

$$
\left\|\left(-A_{w}(t)\right)^{-\theta_{B}} x \sqrt{Q}\right\|_{\gamma\left(L^{2}(S), L^{p}(S)\right)} \lesssim\|\sqrt{Q}\|_{\mathcal{B}\left(L^{2}(S), L^{q}(S)\right)}\|x\|_{L^{p}(S)} .
$$

It follows that there exists a constant $K$ such that for all $x, y \in L^{p}(S)$ and for all $t \in[0, T], \omega \in \Omega$,

$$
\begin{aligned}
& \left\|\left(-A_{w}(t)\right)^{-\theta_{B}}(B(t, \omega, x)-B(t, \omega, y))\right\|_{\gamma\left(L^{2}(S), L^{p}(S)\right)} \leq K\|x-y\|_{L^{p}(S)}, \\
& \left\|\left(-A_{w}(t)\right)^{-\theta_{B}} B(t, \omega, x)\right\|_{\gamma\left(L^{2}(S), L^{p}(S)\right)} \leq K\left(1+\|x\|_{L^{p}(S)}\right) .
\end{aligned}
$$

By Theorem 6.3 (1) we obtain that there exists a unique mild solution $u$ with paths in $C\left([0, T] ; L^{p}(S)\right)$. The fact that $u$ is also the unique variational solution follows in the same way as Example 8.2.

(2) Let $\lambda, \delta>0$ be such that $\lambda+\delta<\beta$. Let $\delta, \lambda>0$ be such that $\delta+\lambda<\beta$. Let $\delta^{\prime}>\delta$ be such that $\delta^{\prime}+\lambda<\beta$. Choose $p \in[2, \infty)$ so large and $\theta_{B}>\frac{n}{2 r}=\frac{n}{2}\left(\frac{1}{p}+\frac{1}{q}\right)$ such that $\beta<\frac{1}{2}-\theta_{B}$.

As in Example 8.2 one has $u_{0} \in E_{\delta^{\prime}+\lambda}^{0}$. By Theorem 6.3 (2) we obtain that $u$ has a version with paths in $C^{\lambda}\left([0, T] ; B_{p, 2}^{2 \delta^{\prime}}(S)\right)$. By [54, Theorem 4.6.1(e)] $B_{p, 2}^{2 \delta^{\prime}}(S) \hookrightarrow$ $C^{2 \delta^{\prime \prime}-\frac{n}{p}}(S)$ where $\delta<\delta^{\prime \prime}<\delta^{\prime}$. Choosing $p$ large enough gives the result.

As a final example we consider again (8.1), but this time with locally Lipschitz coefficients $f$ and $b$.

EXAMPLE 8.9. Consider equation (8.1). Assume that $f, g:[0, T] \times \Omega \times S \times \mathbb{R} \rightarrow$ $\mathbb{R}$ are measurable, adapted and $f$ and $g$ are locally Lipschitz in the fourth variable 
uniform in the others, i.e., for all $R>0$, there exists $L_{f, R}$ and $L_{g, R}$ such that for all $t \in[0, T], \omega \in \Omega, s \in \mathbb{R}$ and $x, y \in \mathbb{R}$ with $|x|,|y| \leq R$,

$$
\begin{gathered}
|f(t, \omega, s, x)-f(t, \omega, s, y)| \leq L_{f, R}|x-y|, t \in[0, T], \omega \in \Omega, s \in S, \\
|g(t, \omega, s, x)-g(t, \omega, s, y)| \leq L_{g, R}|x-y|, t \in[0, T], \omega \in \Omega, s \in S .
\end{gathered}
$$

Assume that $A, C$ and $Q$ are as in Example 8.2. The following statements hold:

(1) Let $p \in(2 n, \infty)$. Let $a \in\left(\frac{n}{p}, \frac{1}{2}\right)$. If $u_{0} \in B_{p, p}^{a}(S)$ a.s., then there exists a unique maximal local mild solution $(u(t))_{t \in[0, \tau)}$ of (8.1) with paths in $C\left([0, \tau) ; B_{p, p}^{2 a}(S)\right)$ a.s.

(2) If $u_{0} \in C^{1}(\bar{S})$ a.s., then the solution $u$ is in $C^{\lambda}\left([0, T] ; C^{2 \delta}(S)\right)$ for all $\lambda, \delta>0$ such that $\lambda+\delta<\frac{1}{2}$. In particular, $u \in C^{\beta_{1}, \beta_{2}}(\bar{S} \times[0, T])$ for all $\beta_{1} \in(0,1)$ and $\beta_{2} \in\left(0, \frac{1}{2}\right)$.

If $f$ and $g$ are also of linear growth, i.e., (8.4) and (8.6), then the following hold:

$(1)^{\prime}$ Let $p \in(2 n, \infty)$. Let $a \in\left(\frac{n}{p}, \frac{1}{2}\right)$. If $u_{0} \in B_{p, p}^{a}(S)$ a.s., then $\tau=T$ and the solution $u$ from above is the unique global mild and variational solution of (8.1) with paths in $C\left([0, T] ; B_{p, p}^{a}(S)\right)$ a.s.

(2) If $u_{0} \in C^{1}(\bar{S})$ a.s., then the solution $u$ is in $C^{\lambda}\left([0, T] ; C^{2 \delta}(S)\right)$ for all $\lambda, \delta>0$ such that $\lambda+\delta<\frac{1}{2}$. In particular, $u \in C^{\beta_{1}, \beta_{2}}(\bar{S} \times[0, T])$ for all $\beta_{1} \in(0,1)$ and $\beta_{2} \in\left(0, \frac{1}{2}\right)$.

REMARK 8.10. (1) If $Q$ is as in Example 8.6, then one can still give conditions under which existence, uniqueness, and regularity hold. This is left to the reader.

(2) It is an interesting question under what conditions on $f$ and $g$ different as (8.4) and (8.6), one still obtains a global solution. There are many results and approaches in this direction. We refer the reader to [33] and references therein. We believe it is important to extend the ideas from [33] to our general framework. This could lead to new global existence results.

We turn to the proof of Example 8.9. The set-up is similar as in Example 8.2, but we need that $a>0$ to be able to consider the locally Lipschitz coefficients $f$ and $b$. Here, $a$ is the parameter from Theorem 7.2 which is used for the underlying space $\widetilde{E}_{a}$. The main reason we want $a>0$ is that $\widetilde{E}_{a} \hookrightarrow C(\bar{S})$ is needed.

Proof of Example 8.9. (1) By [54, Theorem 4.6.1(e)] it follows that $\widetilde{E}_{a} \hookrightarrow C(\bar{S})$ since $a>\frac{n}{p}$. Let $E$ and $A$ be as in Example 8.2. For $0<\eta \leq 1$ let

$$
\widetilde{E}_{\eta}:=\left(L^{p}(S), W^{2, p}(S)\right)_{\eta, p}=B_{p, p}^{2 \eta}(S) .
$$

It follows from [5, Theorem 7.2 and Remark 7.3] and [18, Theorem 2.3] that for $2 \eta \neq \frac{1}{p}$,

$$
E_{\eta}^{t}:=(E, D(A(t)))_{\eta, p} \hookrightarrow \widetilde{E}_{\eta} \hookrightarrow E
$$

with uniform constants in $t \in[0, T]$. Therefore, the version of $(\mathrm{H} 1)_{\eta_{0}}$ explained below (H1) $)_{\eta_{0}}^{\prime}$ in Section 2.1 is satisfied except maybe for $2 \eta=\frac{1}{p}$, but this is 
not an actual problem since we can always take $\eta$ slightly larger in the above arguments. Note that by (8.15) and (8.16), $u_{0} \in E_{a}^{0}$ a.s.

Define $F:[0, T] \times \Omega \times \widetilde{E}_{a} \rightarrow E$ by $F(t, \omega, x)(s)=f(t, \omega, s, x(s))$. By (8.3) and $\widetilde{E}_{a} \hookrightarrow C(\bar{S}), F$ satisfies $(\mathrm{H} 2)^{\prime}$. Let $B:[0, T] \times \Omega \times \widetilde{E}_{a} \rightarrow \gamma\left(L^{2}(S), E\right)$ be defined as

$$
(B(t, \omega, x) h)(s)=b(t, \omega, s, x(s))(\sqrt{Q} h)(s) .
$$

By (8.5), $\widetilde{E}_{a} \hookrightarrow C(\bar{S})$, and the assumptions this is well-defined and it satisfies (H3)'.

By Theorem 7.2 with $\theta_{F}=\theta_{B}=0$, we obtain that there exists a unique mild solution $U$ with paths in $C\left([0, \tau) ; \widetilde{E}_{a}\right)$ a.s.

(2) Let $\lambda, \delta>0$ be such that $\lambda+\delta<\frac{1}{2}$. Let $a>0$ be such that $\lambda+\delta+a<\frac{1}{2}$ and let $p \in[2, \infty)$ be such that $a>\frac{n}{p}$. Let $E$ and $\widetilde{E}_{a}$ and $F, B$ etc. be as in (1). If $u_{0} \in C^{1}(\bar{S})$ a.s., then as before one can show that $u_{0} \in E_{b}^{0}$ a.s. for all $b \in\left[0, \frac{1}{2}\right)$.

By Theorem 7.2 the process $U$ has the following regularity property: $U \in$ $C^{\lambda}\left([0, T] ; \widetilde{E}_{a+\delta}\right)$ a.s. In particular, it follows from [54, Theorem 4.6.1(e)] that $U \in C^{\lambda}\left([0, \tau) ; C^{2 \delta}(S)\right)$ for all $\lambda, \delta>0$ such that $\lambda+\delta<\frac{1}{2}$.

$(1)^{\prime}$ and (2)': This can be proved in the same way as (1) and (2), but now using the linear growth assumption and the last part of Theorem 7.2.

\section{Appendix A. Technical proofs}

Below we prove Proposition 5.4. We recall it for convenience.

PROPOSITION A.1. Assume (AT), (H1) $\eta_{\eta_{0}},(\mathrm{H} 2),(\mathrm{H} 3)$ and $(\mathrm{W})$. Let $r \in(2, \infty)$ be such that $\theta_{B}<\frac{1}{2}-\frac{1}{r}$. Let $U:[0, T] \times \Omega \rightarrow \widetilde{E}_{a}$ be strongly measurable and adapted and such that $U \in L^{r}\left(0, T ; \widetilde{E}_{a}\right)$ a.s. The following assertions are equivalent:

(1) $E$ is a mild solution of (SE).

(2) $U$ is a variational solution of (SE).

Condition (W) from Section 5 is only needed in (2) $\Rightarrow$ (1).

Proof. (1) $\Rightarrow$ (2): Let

$$
F_{-\theta_{F}}(r, x)=\left(-A_{w}(r)\right)^{-\theta_{F}} F(r, x), \quad B_{-\theta_{B}}(r, x)=\left(-A_{w}(r)\right)^{-\theta_{B}} B(r, x)
$$

and $P_{\theta}(t, r)=P(t, r)\left(-A_{w}(r)\right)^{\theta}$ for $\theta=\theta_{F}$ or $\theta=\theta_{B}$.

Let $t \in[0, T]$ be arbitrary and $\varphi \in \Gamma_{t}$. Since $U$ is a.s. in $L^{1}(0, T ; E)$ we have that $s \mapsto\left\langle U(s), A(s)^{*} \varphi(s)\right\rangle$ is integrable and from the definition of a mild solution we obtain that a.s., 


$$
\begin{aligned}
& \int_{0}^{t}\left\langle U(s), A(s)^{*} \varphi(s)\right\rangle \mathrm{d} s \\
& =\int_{0}^{t}\left\langle P(s, 0) u_{0}, A(s)^{*} \varphi(s)\right\rangle \mathrm{d} s+\int_{0}^{t} \int_{0}^{s}\left\langle P_{\theta_{F}}(s, r) F_{-\theta_{F}}(r, U(r)), A(s)^{*} \varphi(s)\right\rangle \mathrm{d} r \mathrm{~d} s \\
& \quad+\int_{0}^{t} \int_{0}^{s} B_{-\theta_{B}}(r, U(r))^{*} P_{\theta_{B}}(s, r)^{*} A(s)^{*} \varphi(s) \mathrm{d} W_{H}(r) \mathrm{d} s .
\end{aligned}
$$

Since $(P(t, s))_{0 \leq s \leq t \leq T}$ is an evolution family that solves (2.1), it follows from an approximation argument that for all $x \in E$ and $0 \leq r \leq t \leq T$,

$$
\begin{aligned}
& \langle P(t, r) x, \varphi(t)\rangle-\langle x, \varphi(r)\rangle \\
& \quad=\int_{r}^{t}\left\langle P(s, r) x, A(s)^{*} \varphi(s)\right\rangle \mathrm{d} s+\int_{r}^{t}\left\langle P(s, r) x, \varphi^{\prime}(s)\right\rangle \mathrm{d} s .
\end{aligned}
$$

Therefore, by another approximation argument we obtain that for all $\theta \in[0,1)$ and for all $x \in E$ and $0 \leq r \leq t$,

$$
\begin{aligned}
& \left\langle P_{\theta}(t, r) x, \varphi(t)\right\rangle-\left\langle x,\left(\left(-A_{w}(r)\right)^{\theta}\right)^{*} \varphi(r)\right\rangle \\
& \quad=\int_{r}^{t}\left\langle P_{\theta}(s, r) x, A(s)^{*} \varphi(s)\right\rangle \mathrm{d} s+\int_{r}^{t}\left\langle P_{\theta}(s, r) x, \varphi^{\prime}(s)\right\rangle \mathrm{d} s .
\end{aligned}
$$

As a consequence one obtains that for all $R \in \mathcal{B}(H, E)$ and $0 \leq r \leq t$,

$$
\begin{aligned}
R^{*} & P_{\theta_{B}}(t, r)^{*} \varphi(t)-R^{*}\left(\left(-A_{w}(r)\right)^{\theta_{B}}\right)^{*} \varphi(r) \\
= & \int_{r}^{t} R^{*} P_{\theta_{B}}(s, r)^{*} A(s)^{*} \varphi(s) \mathrm{d} s+\int_{r}^{t} R^{*} P_{\theta_{B}}(s, r)^{*} \varphi^{\prime}(s) \mathrm{d} s .
\end{aligned}
$$

Indeed, this follows from (A.3) by applying $h \in H$ on both sides.

By the Fubini theorem and (A.3) we obtain a.s.,

$$
\begin{aligned}
\int_{0}^{t} & \int_{0}^{s}\left\langle P_{\theta_{F}}(s, r) F_{-\theta_{F}}(r, U(r)), A(s)^{*} \varphi(s)\right\rangle \mathrm{d} r \mathrm{~d} s \\
= & \int_{0}^{t}\left\langle P_{\theta_{F}}(t, r) F_{-\theta_{F}}(r, U(r)), \varphi(t)\right\rangle \mathrm{d} r-\int_{0}^{t}\left\langle F_{-\theta_{F}}(r, U(r)),\left(\left(-A_{w}(r)\right)^{\theta_{F}}\right)^{*} \varphi(r)\right\rangle \mathrm{d} r \\
& -\int_{0}^{t} \int_{0}^{s}\left\langle P_{\theta_{F}}(s, r) F_{-\theta_{F}}(r, U(r)), \varphi^{\prime}(s)\right\rangle \mathrm{d} r \mathrm{~d} s .
\end{aligned}
$$

By the stochastic Fubini theorem and (A.4) we obtain that a.s.,

$$
\begin{aligned}
\int_{0}^{t} & \int_{0}^{s} B_{-\theta_{B}}(r, U(r))^{*} P_{\theta_{B}}(s, r)^{*} A(s)^{*} \varphi(s) \mathrm{d} W_{H}(r) \mathrm{d} s \\
= & \int_{0}^{t} B_{-\theta_{B}}(r, U(r))^{*} P_{\theta_{B}}(t, r)^{*} \varphi(t) \mathrm{d} W_{H}(r) \\
& -\int_{0}^{t} B_{-\theta_{B}}(r, U(r))^{*}\left(\left(-A_{w}(r)\right)^{\theta_{B}}\right)^{*} \varphi(r) \mathrm{d} W_{H}(r) \\
& -\int_{0}^{t} \int_{r}^{t} B_{-\theta_{B}}(r, U(r))^{*} P_{\theta_{B}}(s, r)^{*} \varphi^{\prime}(s) \mathrm{d} W_{H}(r) \mathrm{d} s .
\end{aligned}
$$


Therefore, it follows from (A.1), (A.2) and the definition of a mild solution that

$$
\begin{aligned}
\int_{0}^{t}\left\langle U(s), A(s)^{*} \varphi(s)\right\rangle \mathrm{d} s= & \langle U(t), \varphi(t)\rangle-\int_{0}^{t}\left\langle U(s), \varphi^{\prime}(s)\right\rangle \mathrm{d} s-\left\langle u_{0}, \varphi(0)\right\rangle \\
& -\int_{0}^{t}\left\langle F_{-\theta_{F}}(r, U(r)),\left(\left(-A_{w}(r)\right)^{\theta_{F}}\right)^{*} \varphi(r)\right\rangle \mathrm{d} r \\
& -\int_{0}^{t} B_{-\theta_{B}}(r, U(r))\left(\left(-A_{w}(r)\right)^{\theta_{B}}\right)^{*} \varphi(r) \mathrm{d} W_{H}(r)
\end{aligned}
$$

and we obtain that $U$ is a variational solution.

$(2) \Rightarrow(1)$ : Let $t \in[0, T]$ be arbitrary. We show that for all $x^{*} \in \Upsilon_{t}$, a.s.

$$
\begin{gathered}
\left\langle U(t), x^{*}\right\rangle=\left\langle P(t, 0) u_{0}, x^{*}\right\rangle+\int_{0}^{t}\left\langle P_{\theta_{F}}(t, s) F_{-\theta_{F}}(s, U(s)), x^{*}\right\rangle \mathrm{d} s \\
\left.+\int_{0}^{t} B_{-\theta_{B}}(s, U(s))\right)^{*} P_{\theta_{B}}(t, s)^{*} x^{*} \mathrm{~d} W_{H}(s) .
\end{gathered}
$$

By the existence of the integral, the existence of the stochastic integral, the weak*sequential density of $\Upsilon_{t}$ (see (W) and the Hahn-Banach theorem this suffices. For $x^{*} \in \Upsilon_{t}$, let $\varphi(s)=P(t, s)^{*} x^{*}$. Then it follows from (5.5) and (5.6) that

$$
\begin{aligned}
& \left\langle U(t), x^{*}\right\rangle-\left\langle P(t, 0) u_{0}, x^{*}\right\rangle+\int_{0}^{t}\left\langle U(s), A(s)^{*} P(t, s)^{*} x^{*}\right\rangle \mathrm{d} s \\
& =\int_{0}^{t}\left\langle U(s), A(s)^{*} P(t, s)^{*} x * \mathrm{~d} s+\int_{0}^{t}\left\langle F_{-\theta_{F}}(s, U(s)),\left(\left(-A_{w}(s)\right)^{\theta_{F}}\right)^{*} P(t, s)^{*} x^{*}\right\rangle \mathrm{d} s\right. \\
& \quad+\int_{0}^{t} B_{-\theta_{B}}(s, U(s))^{*}\left(\left(-A_{w}(s)\right)^{\theta_{B}}\right)^{*} P(t, s)^{*} x^{*} \mathrm{~d} W_{H}(s)
\end{aligned}
$$

and we may conclude (A.5).

\section{Acknowledgements}

The author is grateful to Roland Schnaubelt and Lutz Weis for helpful discussions. Moreover, he thanks the anonymous referees for carefully reading the manuscript and for giving many useful comments.

Open Access. This article is distributed under the terms of the Creative Commons Attribution Noncommercial License which permits any noncommercial use, distribution, and reproduction in any medium, provided the original author(s) and source are credited.

\section{REFERENCES}

[1] P. ACQUISTAPACE, Evolution operators and strong solutions of abstract linear parabolic equations, Differential Integral Equations 1 (1988), no. 4, 433-457.

[2] P. ACQUISTAPACE AND B. TERRENI, A unified approach to abstract linear nonautonomous parabolic equations, Rend. Sem. Mat. Univ. Padova 78 (1987), 47-107. 
[3] P. ACQUiSTAPACE AND B. TeRRENI, Regularity properties of the evolution operator for abstract linear parabolic equations, Differential Integral Equations 5 (1992), no. 5, 1151-1184.

[4] D. Albrecht, X. Duong, And A. McIntosh, Operator theory and harmonic analysis, Instructional Workshop on Analysis and Geometry, Part III (Canberra, 1995), Proc. Centre Math. Appl. Austral. Nat. Univ., vol. 34, Austral. Nat. Univ., Canberra, 1996, pp. 77-136.

[5] H. AmanN, Nonhomogeneous linear and quasilinear elliptic and parabolic boundary value problems, Function spaces, differential operators and nonlinear analysis (Friedrichroda, 1992), Teubner-Texte Math., vol. 133, Teubner, Stuttgart, 1993, pp. 9-126.

[6] H. Amann, Linear and quasilinear parabolic problems. Vol. I, Abstract linear theory, Monographs in Mathematics, vol. 89, Birkhäuser Boston Inc., Boston, MA, 1995.

[7] V. I. BogAchev, Gaussian measures, Mathematical Surveys and Monographs, vol. 62, American Mathematical Society, Providence, RI, 1998.

[8] Z. BRZEŹNIAK, Stochastic partial differential equations in M-type 2 Banach spaces, Potential Anal. 4 (1995), no. 1, 1-45.

[9] Z. BRZEŹNIAK, On stochastic convolution in Banach spaces and applications, Stochastics Stochastics Rep. 61 (1997), no. 3-4, 245-295.

[10] Z. BRZEŹNIAK, B. MASLOWSKI, AND J. SEIDLER, Stochastic nonlinear beam equations, Probab. Theory Related Fields 132 (2005), no. 1, 119-149.

[11] Z. BRZEŹNIAK AND J.M.A.M. VAN NEERVEN, Space-time regularity for linear stochastic evolution equations driven by spatially homogeneous noise, J. Math. Kyoto Univ. 43 (2003), no. 2, 261-303.

[12] D. L. BURKHOLDER, Martingales and singular integrals in Banach spaces, Handbook of the geometry of Banach spaces, Vol. I, North-Holland, Amsterdam, 2001, pp. 233-269.

[13] S. CERRAI, Stochastic reaction-diffusion systems with multiplicative noise and non-Lipschitz reaction term, Probab. Theory Related Fields 125 (2003), no. 2, 271-304.

[14] G. DA PRATO, S. KWAPIEŃ, AND J. ZABCZYK, Regularity of solutions of linear stochastic equations in Hilbert spaces, Stochastics 23 (1987), no. 1, 1-23.

[15] G. Da PRATO AND J. ZABCZYK, A note on stochastic convolution, Stochastic Anal. Appl. 10 (1992), no. 2, 143-153.

[16] G. DA PRATO AND J. ZABCZYK, Stochastic equations in infinite dimensions, Encyclopedia of Mathematics and its Applications, vol. 44, Cambridge University Press, Cambridge, 1992.

[17] D. A. DAWSON, Stochastic evolution equations and related measure processes, J. Multivariate Anal. 5 (1975), 1-52.

[18] R. Denk, G. Dore, M. Hieber, J. Pruss, And A. Venni, New thoughts on old results of R. T. Seeley, Math. Ann. 328 (2004), no. 4, 545-583.

[19] J. DetTweILER, J.M.A.M. VAN NEERVEN, AND L. W. WeIs, Space-time regularity of solutions of parabolic stochastic evolution equations, Stoch. Anal. Appl. 24 (2006), 843-869.

[20] J. Diestel, H. Jarchow, AND A. TONGE, Absolutely summing operators, Cambridge Studies in Advanced Mathematics, vol. 43, Cambridge University Press, Cambridge, 1995.

[21] M. HAASE, The functional calculus for sectorial operators, Operator Theory: Advances and Applications, vol. 169, Birkhäuser Verlag, Basel, 2006.

[22] N. J. KAlton AND L. W. WeIs, The $H^{\infty}$-calculus and square function estimates, Preprint, 2004.

[23] T. KATO, Remarks on pseudo-resolvents and infinitesimal generators of semi-groups, Proc. Japan Acad. 35 (1959), 467-468.

[24] N. V. KRYLOV, An analytic approach to SPDEs, Stochastic partial differential equations: six perspectives, Math. Surveys Monogr., vol. 64, Amer. Math. Soc., Providence, RI, 1999, pp. $185-242$.

[25] N. V. KRYlov AND B. L. Rozovski, Stochastic evolution equations, Current problems in mathematics, Vol. 14 (Russian), Akad. Nauk SSSR, Vsesoyuz. Inst. Nauchn. i Tekhn. Informatsii, Moscow, 1979, pp. 71-147, 256.

[26] P. C. KUnSTMANN AND L. W. WeIS, Maximal $L_{p}$-regularity for parabolic equations, Fourier multiplier theorems and $H^{\infty}$-functional calculus, Functional analytic methods for evolution equations, Lecture Notes in Math., vol. 1855, Springer, Berlin, 2004, pp. 65-311. 
[27] C. LE MERDY, The Weiss conjecture for bounded analytic semigroups, J. London Math. Soc. (2) 67 (2003), no. 3, 715-738.

[28] J.- L. LIONS, Quelques méthodes de résolution des problèmes aux limites non linéaires, Dunod, 1969.

[29] A. LUNARDI, On the evolution operator for abstract parabolic equations, Israel J. Math. 60 (1987), no. 3, 281-314.

[30] A. LUNARDI, Analytic semigroups and optimal regularity in parabolic problems, Progress in Nonlinear Differential Equations and their Applications, 16, Birkhäuser Verlag, Basel, 1995.

[31] L. MANIAR AND R. SChNAUBELT, The Fredholm alternative for parabolic evolution equations with inhomogeneous boundary conditions, J. Differential Equations 235 (2007), no. 1, 308-339.

[32] L. MANIAR AND R. SCHNAUBELT, Robustness of Fredholm properties of parabolic evolution equations under boundary perturbations, J. Lond. Math. Soc. (2) 77 (2008), no. 3, 558-580.

[33] R. MANTHEY AND T. ZAUSINGER, Stochastic evolution equations in $L_{\rho}^{2 v}$, Stochastics Stochastics Rep. 66 (1999), no. 1-2, 37-85.

[34] A. McInTosh, Operators which have an $H_{\infty}$ functional calculus, Miniconference on operator theory and partial differential equations (North Ryde, 1986), Proc. Centre Math. Anal. Austral. Nat. Univ., vol. 14, Austral. Nat. Univ., Canberra, 1986, pp. 210-231.

[35] A. McInTOSH AND A. YAGI, Operators of type $\omega$ without a bounded $H_{\infty}$ functional calculus, Miniconference on Operators in Analysis (Sydney, 1989), Proc. Centre Math. Anal. Austral. Nat. Univ., vol. 24, Austral. Nat. Univ., Canberra, 1990, pp. 159-172.

[36] J.M.A.M. VAN NEERVEN, M.C. VERAAR, AND L. WeIS, Stochastic integration in UMD Banach spaces, Ann. Probab. 35 (2007), no. 4, 1438-1478.

[37] J.M.A.M. VAN NEERVEN, M.C. VERAAR, AND L.W. WEIS, Stochastic evolution equations in UMD Banach spaces, J. Functional Anal. 255 (2008), 940-993.

[38] J.M.A.M. VAN NEERVEN AND L.W. WEIS, Stochastic integration of functions with values in a Banach space, Studia Math. 166 (2005), no. 2, 131-170.

[39] G. NICKEL, On evolution semigroups and wellposedness of nonautonomous Cauchy problems. Ph.D. thesis, Tübingen: Univ. Tübingen, Math. Fak. 91 S., 1996.

[40] G. NiCKEL, Evolution semigroups for nonautonomous Cauchy problems, Abstr. Appl. Anal. 2 (1997), no. 1-2, 73-95.

[41] E. PARDouX, Équations aux dérivées partielles stochastiques nonlinéares monotones: étude de solutions fortes de type Itô., Ph.D. thesis, Université Paris-Orsay, 1975.

[42] E. PARDOUX, Stochastic partial differential equations and filtering of diffusion processes, Stochastics 3 (1979), no. 2, 127-167.

[43] A. PAZY, Semigroups of linear operators and applications to partial differential equations, Applied Mathematical Sciences, vol. 44, Springer-Verlag, New York, 1983.

[44] G. PISIER, Martingales with values in uniformly convex spaces, Israel J. Math. 20 (1975), no. 3-4, 326-350.

[45] B.L. RozovsKi, Stochastic evolution systems, Mathematics and its Applications (Soviet Series), vol. 35, Kluwer Academic Publishers Group, Dordrecht, 1990, Linear theory and applications to nonlinear filtering, Translated from the Russian by A. Yarkho.

[46] M. SANZ- SOlÉ AND P.-A. VuILLERMOT, Hölder-Sobolev regularity of solutions to a class of SPDE's driven by a spatially colored noise, C. R. Math. Acad. Sci. Paris 334 (2002), no. 10, 869-874.

[47] M. SANZ-SOlÉ AND P.-A. VUILlERMOT, Equivalence and Hölder-Sobolev regularity of solutions for a class of non-autonomous stochastic partial differential equations, Ann. Inst. H. Poincaré Probab. Statist. 39 (2003), no. 4, 703-742.

[48] R. SchNAUBELT, Asymptotic behaviour of parabolic nonautonomous evolution equations, Functional analytic methods for evolution equations, Lecture Notes in Math., vol. 1855, Springer, Berlin, 2004, pp. 401-472.

[49] R. SCHNAUBELT AND M.C. VERAAR, Stochastic equations with boundary noise, In preparation., 2008.

[50] R. SEELEY, Interpolation in $L^{p}$ with boundary conditions, Studia Math. 44 (1972), 47-60, Collection of articles honoring the completion by Antoni Zygmund of 50 years of scientific activity, I. 
[51] J. SEIDLER, Da Prato-Zabczyk's maximal inequality revisited. I, Math. Bohem. 118 (1993), no. 1, 67-106.

[52] H. TAnabe, Equations of evolution, Monographs and Studies in Mathematics, vol. 6, Pitman (Advanced Publishing Program), Boston, Mass., 1979.

[53] H. TANABE, Functional analytic methods for partial differential equations, Monographs and Textbooks in Pure and Applied Mathematics, vol. 204, Marcel Dekker Inc., New York, 1997.

[54] H. TRIEBEL, Interpolation theory, function spaces, differential operators, second ed., Johann Ambrosius Barth, Heidelberg, 1995.

[55] M.C. VERAAR, Stochastic Integration in Banach spaces and Applications to Parabolic Evolution Equations, Ph.D. thesis, Delft University of Technology, 2006, http://fa.its.tudelft.nl/ veraar/.

[56] M.C. VERAAR AND J. ZIMMERSCHIED, Non-autonomous stochastic Cauchy problems in Banach spaces, Studia Math. 185 (2008), no. 1, 1-34.

[57] A. YAGI, Parabolic evolution equations in which the coefficients are the generators of infinitely differentiable semigroups. II, Funkcial. Ekvac. 33 (1990), no. 1, 139-150.

[58] A. YAGI, Abstract quasilinear evolution equations of parabolic type in Banach spaces, Boll. Un. Mat. Ital. B (7) 5 (1991), no. 2, 341-368.

[59] K. YosIdA, Functional analysis, Classics in Mathematics, Springer-Verlag, Berlin, 1995, Reprint of the sixth (1980) edition.

[60] X. ZHANG, $L^{p}$-theory of semi-linear SPDEs on general measure spaces and applications, J. Funct. Anal. 239 (2006), no. 1, 44-75.

\author{
M. C. Veraar \\ Delft Institute of Applied Mathematics, \\ Delft University of Technology, \\ P.O. Box 5031, \\ 2600 GA Delft, \\ The Netherlands \\ E-mail:mark@profsonline.nl; M.C.Veraar@tudelft.nl
}

